\title{
RDUS
}

Revue de DROIT UNIVERSITÉ DE SHERBROOKE

Titre : $\quad$ L'ENDETTEMENT DE L'ENTREPRISE AU QUÉBEC : PARAMÈTRES JURIDIQUES

Auteur(s) : $\quad$ Vilaysoun LOUNGNARATH, jr

Revue : $\quad$ RDUS, 1995-1996, volume 26, numéro 1

Pages: $\quad 1-44$

ISSN : $\quad 0317-9656$

Éditeur : $\quad$ Université de Sherbrooke. Faculté de droit.

URI : $\quad$ http://hdl.handle.net/11143/12856

DOI : https://doi.org/10.17118/11143/12856 
Page vide laissée intentionnellement. 


\title{
ARTICLE
}

\section{L'ENDETTEMENT DE L'ENTREPRISE AU QUÉBEC : PARAMĖTRES JURIDIQUES}

\author{
par Vilaysoun LOUNGNARATH, jr ${ }^{* *}$
}

Privilégiant une approche synthétique, l'auteur cherche à cerner les principaux paramètres juridiques de l'endettement de l'entreprise au Québec. Il s'intéresse d'abord au contrôle légal et judiciaire de l'endettement de l'entreprise. Puis, il livre quelques réflexions sur le domaine de la notion juridique de dette, en insistant sur la décision de la Cour suprême du Canada dans l'affaire Société d'assurance-dépôts du Canada $c$. Banque Commerciale Canadienne. Trois autres thèmes sont abordés : l'endettement d'un groupe de sociétés, les sûretés générales conventionnelles et le redressement de l'entreprise excessivement endettée.

Through a synthetic approach, the writer sets out the main legal parameters of corporate debt in Quebec. First, the legal regulation of corporate debt is broadly examined. In the second part, some remarks are made on the extension of the legal notion of corporate debt, with an emphasis on the Supreme Court decision in Canada Deposit Insurance Corp. v. Canadian Commercial Bank. Three other topics are discussed : the debt of partnerships or of corporate entities, contractual general securities and the reorganization of financially distressed businesses.

1. La présente étude est basée sur un rapport qui a été présenté aux Journées internationales de l'Association Henri Capitant des amis de la culture juridique française, à Buenos Aires, en 1995.

2. Professeur à la Faculté de droit de l'Université de Montréal; membre du Barreau du Québec et du Barreau de l'état de New York. L'auteur tient à remercier Monsieur Rudi Arès pour son travail d'assistance de recherche. 


\section{SOMMAIRE}

INTRODUCTION

I. Le contrôle légal et judiciaire de l'endettement de l'entreprise . 6

A- Les limites juridiques à la capacité d'endettement : contraintes extrinsèques à l'entreprise $\ldots \ldots \ldots \ldots \ldots \ldots \ldots \ldots \ldots$

1- Une politique de non-interventionnisme dans la capitalisation de l'entreprise .........................6 6

2- Un contrôle indirect de l'endettement de l'entreprise via la réglementation prudentielle et la surveillance des institutions financières $\ldots \ldots \ldots \ldots \ldots \ldots \ldots \ldots \ldots \ldots \ldots$

B- Les mécanismes de contrôle de l'endettement intrinsèques à la société par actions ........................ 9

1- L'organisation du pouvoir d'emprunter et de consentir une sûreté dans la société par actions . ............. 9

2- L'actionnaire et l'endettement de l'entreprise dans le schéma du droit commun ........................ 10

3- Le recours statutaire pour oppression de l'actionnaire et l'endettement de l'entreprise .................. 11

C- La responsabilité civile du prêteur eu égard à la décision d'octroyer un crédit ........................... 13

D- Les limites juridiques relatives aux modalités et aux conditions de l'endettement de l'entreprise ..................... 14

II- Le domaine de la notion juridique de dette $\ldots \ldots \ldots \ldots \ldots$

A- Qualification juridique de l'apport externe : dette ou capitaux

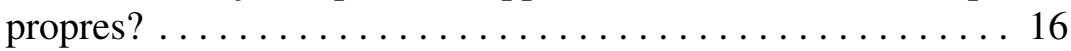

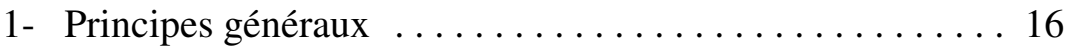

2- La décision Société d'assurance-dépôts du Canada c. Banque commerciale canadienne ................. 18

B- Le traitement comptable et le processus de qualification . . . . 20

C- La thèse de l' «equitable investment» et l'article 139 de la Loi sur la

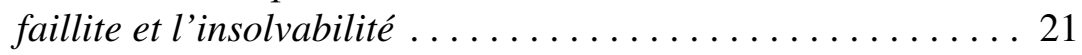

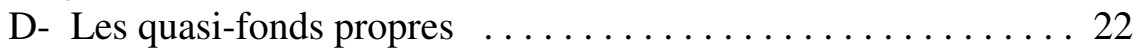




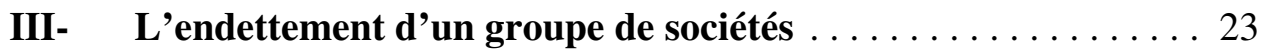

A- L'hégémonie de la doctrine du voile corporatif . . . . . . . 23

B- La «subordination reconnue en equity» ............ 25

IV- Les sûretés générales conventionnelles $\ldots \ldots \ldots \ldots \ldots 26$

A- Le Code civil du Québec et les sûretés générales conventionnelles

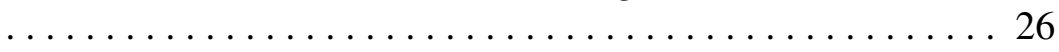

B- Les sûretés de la Loi sur les banques . . . . . . . . . 30

V- Le redressement de l'entreprise excessivement endettée . . . . 31

A- La réalisation des sûretés et la théorie de l'abus des droits contractuels ............................... 31

B- La technique de l' «in-substance defeasance» .......... 32

C- La procédure concordataire de la Loi sur la faillite et l'insolvabilité ............................. 34

D- Le redressement de l'entreprise à l'extérieur de la faillite : la Loi sur les arrangements avec les créanciers des compagnies . . . . . 37

E- L'arrangement de l'article 192 de la Loi sur les sociétés par actions

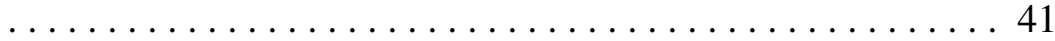

F- L'action privilégiée dite «de détresse» $\ldots \ldots \ldots \ldots \ldots \ldots 42$

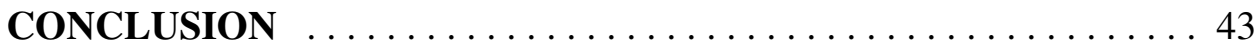





\section{INTRODUCTION}

Au nord du $45^{\mathrm{e}}$ parallèle, les gouvernements ne sont pas les seuls à être endettés. En effet, le poids de l'endettement dans le bilan de l'entreprise canadienne s'est accru sensiblement au cours de ces dernières années. Le ratio des dettes à l'avoir pour l'ensemble des entreprises canadiennes est ainsi passé de moins de 1.00 en 1987 à plus de 1.2 en 1992, pour osciller, depuis, autour de cette valeur. Certes, les effusions boursières du milieu de la décennie quatre-vingt expliquent en partie les ratios d'endettement peu élevés pour l'entreprise canadienne de cette période. Néanmoins, une tendance de fond se dessine : les ratios d'endettement de l'entreprise canadienne se maintiennent, depuis 1992, à des niveaux supérieurs à ce qui avait été enregistré au tout début de la décennie précédente. ${ }^{1}$ Non pas que ce tableau témoigne d'un malaise ou d'un vent de prodigalité : l'emprunt employé aux fins de moderniser l'usine, d'améliorer le réseau de distribution, de soutenir la croissance des ventes ou d'intégrer de nouvelles technologies doit bien avoir quelques vertus pour l'entreprise et la société en général. Ces quelques chiffres ont cependant le mérite de révéler l'actualité du thème de l'encadrement juridique de l'endettement de l'entreprise, objet de la présente étude.

Les paramètres juridiques de l'endettement de l'entreprise appartiennent à trois sous-systèmes : d'abord, l'ensemble des règles se rapportant au financement par emprunt et aux sûretés propres à l'entreprise; ensuite, le droit des sociétés ${ }^{2}$ enfin, les lois régissant la faillite et l'insolvabilité. Au coeur de ces trois ordres juridiques se trouvent deux instruments relativement nouveaux : le

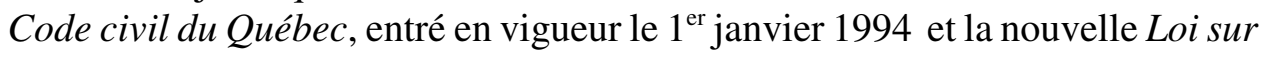
la faillite et l'insolvabilité, adoptée en 1992.

Cette étude propose une vue d'ensemble de l'encadrement juridique de l'endettement de l'entreprise au Québec. Elle est divisée en cinq grandes

1. STATISTIQUES CANADA, «Statistiques financières trimestrielles des entreprises», numéro 61-008 au catalogue, 1994, p. 19; voir aussi R.A. BREALEY, S.C. MYERS et P. LAROCHE, Principes de gestion financière des sociétés, 2e édition, Saint-Laurent, McGraw-Hill, 1992, (traduction) pp. 515 à 520.

2. Cette étude s'intéresse essentiellement à l'entreprise constituée en société par actions ou, pour employer le synonyme québécois, en compagnie. Par ailleurs, nous privilégierons l'examen de la législation fédérale sur les sociétés par actions. 
parties : 1) le contrôle légal et judiciaire de l'endettement de l'entreprise; 2) le domaine de la notion juridique de dette; 3) l'endettement du groupe de sociétés; 4) les sûretés générales conventionnelles; 5) le redressement de l'entreprise excessivement endettée.

\section{Le contrôle légal et judiciaire de l'endettement de l'entreprise}

\section{A- Les limites juridiques à la capacité d'endettement : contraintes extrinsèques à l'entreprise}

\section{1- Une politique de non-interventionnisme dans la capitalisation de l'entreprise}

Au Québec, l'État s'est abstenu de fixer des limites quantitatives au niveau de l'endettement de l'entreprise industrielle ou commerciale. Il n'a pas non plus prescrit dans ses lois des critères qualitatifs ou des procédures d'examen visant à contrôler le volume d'endettement de l'entreprise. Cette forme de non-interventionnisme n'a rien d'étonnant : le Québec est une société dont les usages et traditions commerciaux restent fortement influencés par les institutions du capitalisme nord-américain, dont la culture dominante est marquée par de fortes réticences à l'égard de toute manifestation interventionniste dans les fonctions internes de l'entreprise. ${ }^{3}$ Les législateurs québécois et fédéral estiment que le cadre d'un marché libre, où interagissent

3. Si cette culture est dominante chez le puissant voisin du Sud, elle ne recueille tout de même pas un assentiment unanime. Ainsi, l'économiste américain John Kenneth Galbraith souligne que la vague d'offres publiques d'achat inamicales et de prises de contrôle financées par endettement, qui a déferlé sur les États-Unis dans les années 80, a acculé à la banqueroute un nombre significatif d'entreprises pourtant saines et bien établies; selon l'universitaire, les dégâts auraient été moindres si l'État avait instauré des contrôles légaux ou administratifs de l'endettement. Dans La république des satisfaits, la culture du contentement aux États-Unis (J.K. GALBRAITH, La république des satisfaits, la culture du contentement aux États-Unis, Paris, Éditions du Seuil, 1993, à la page 174), le réputé pamphlétaire s'exprime comme suit : «La grande folie des fusions et achats d'entreprises des années quatre-vingt aurait pu être étouffée dans l'oeuf par une législation qui aurait exigé des auditions et une période d'attente pour toute substitution massive d'une dette à des fonds propres caractéristique universelle du raid boursier et autres fusions et OPA. L'effet restrictif que la dette et les paiements d'intérêt ont exercé sur l'investissement et la productivité de tant de sociétés et les multiples faillites qui ont été enregistrées auraient ainsi été évitées au moindre coût.» 
prêteurs, investisseurs et entreprises à la recherche de leur meilleur intérêt individuel, est «sociétalement» préférable à l'application d'une norme étatique pour l'élaboration de la structure du capital. D'ailleurs, la structure du capital idéale pour une entreprise dépend des circonstances propres à chaque espèce; une approche pragmatique, souple, est toute indiquée et, à ce titre, la supériorité du marché sur le dirigisme apparaît difficilement contestable. Qui plus est, les théoriciens n'ont pas, jusqu'à présent, esquissé de modèle satisfaisant susceptible de servir d'assises à une norme générale convenant à une pluralité de contextes d'affaires. ${ }^{4}$ Le non-interventionnisme se justifie aussi par l'hypothèse que l'entreprise dispose de l'expertise et de l'expérience pour négocier avec ses banquiers et évaluer les conséquences de choix financiers, de même que les risques associés à ces choix; dans la même veine, on plaide qu'il ne relève pas de la mission de la puissance publique de protéger l'entreprise contre sa propre bêtise, son manque de jugement ou sa témérité. De plus, des limites rigides au financement par voie d'emprunt pourraient décourager l'esprit d'initiative et nuire à l'expansion de la petite et moyenne entreprise québécoise, celle-ci disposant d'un accès limité au capital de risque. Enfin, les législateurs sont sans doute confortés dans leurs choix par le conservatisme notoire des banques, partiellement explicable par la structure fortement oligopolistique du système bancaire canadien.

\section{2- Un contrôle indirect de l'endettement de l'entreprise via la réglementation prudentielle et la surveillance des institutions financières}

Les lois du capitalisme ne s'appliquent pas dans toute leur rigueur et leur amplitude aux institutions financières québécoises et canadiennes. En effet, celles-ci évoluent à l'intérieur de cadres réglementaires, somme toute assez exigeants, et leurs activités sont supervisées par diverses autorités administratives. La plus importante d'entre elles est un organisme fédéral, le Bureau du surintendant des institutions financières. Le choix de réglementer les institutions financières s'explique notamment par le rôle crucial joué par celles-ci dans l'intermédiation des capitaux, une fonction névralgique dans l'économie d'un pays. Elle tient aussi à la volonté de l'État de contenir les effets pervers de la structure oligopolistique du système financier canadien; car toute 
structure oligopolistique a le défaut de concentrer le pouvoir, ce qui peut éventuellement se traduire par une diminution du bien-être collectif, des inégalités économiques et une baisse de la qualité de la vie démocratique.

Par le jeu du cadre réglementaire des institutions financières, l'endettement de l'entreprise se trouve à être indirectement soumis à une forme de contrôle étatique. Ainsi, selon l'article 465 de la Loi sur les banques, le conseil d'administration d'une banque a le devoir de gérer le portefeuille de placements et de prêts de la banque selon des principes, normes et procédures établis sur le modèle de la personne prudente. ${ }^{5}$

La directive B $1,{ }^{6}$ datée de janvier 1993, met en relief des facteurs que la direction et le conseil d'administration d'une institution financière de régime fédéral devraient considérer dans l'élaboration et la mise en oeuvre des politiques, normes et procédures relatives à ses investissements et à ses prêts. Il ressort clairement de la directive B1 que la norme de la personne prudente et raisonnable se rapporte à la gestion de l'agrégat des actifs financiers; d'ordre macroscopique, cette norme devrait toutefois, en toute logique, se répercuter sur le rapport particulier entre l'institution prêteuse et l'entreprise.

Dans le même ordre d'idées, signalons que le Bureau du surintendant des institutions financières a édicté la directive $\mathrm{B} 2$, un texte normatif sur les prêts d'envergure. La directive $\mathrm{B} 2^{7} \mathrm{~s}$ 'adresse aux banques à charte et pose notamment que, sauf exception spécifiquement prévue, le risque total assuré en regard d'un emprunteur donné ne doit pas excéder vingt-cinq pour cent du capital total de la banque.

5. Loi sur les banques, L.C. 1991, c. 46 [ci-après L.B.].

6. Directive B1 reproduite dans TORY TORY DESLAURIERS \& BINNINGTON, Consolidated Bank Act and Regulations 1994, 6 éd. Scarborough, Carswell, 1994, pp. 432-436 (janvier 1993).

7. Ibid., pp. 437-440. 


\section{B- Les mécanismes de contrôle de l'endettement intrinsèques à la société par actions}

\section{1- L'organisation du pouvoir d'emprunter et de consentir une sûreté dans la société par actions}

En principe, le conseil d'administration de la société de régime fédéral n'a pas à obtenir l'autorisation des actionnaires pour contracter un emprunt ou grever d'une sûreté les biens de la société. ${ }^{8}$ L'assentiment des actionnaires ne sera requis que s'il est prescrit par une disposition des statuts constitutifs, des règlements de la société ou d'une convention unanime des actionnaires. ${ }^{9}$ La Loi sur les compagnies du Québec ${ }^{10}$ retient un système différent : tout comme dans le schéma de la L.S.A., le pouvoir d'emprunt et de consentir des sûretés est exercé par le conseil d'administration et mis en oeuvre individuellement pour chaque cas par l'adoption d'une résolution; ce pouvoir doit cependant s'appuyer sur un règlement général approuvé par les actionnaires. ${ }^{11}$

Une alternative s'offre à la société de régime québécois. En effet, le pouvoir d'emprunter et de consentir des sûretés peut être conféré au conseil d'administration par l'insertion d'une disposition habilitante dans les lettres patentes ${ }^{12}$ ou dans les statuts constitutifs, ${ }^{13}$ ce qui est la règle en pratique. ${ }^{14}$

La L.S.A. et la L.C.Q. autorisent toutes deux le conseil d'administration à déléguer à des dirigeants ou des administrateurs particuliers le pouvoir d'emprunter et de consentir des sûretés.

8. Loi sur les sociétés par actions, L.R.C. (1985), c. C-44, art. 189 [ci-après L.S.A.].

9. Ibid.

10. Loi sur les compagnies du Québec, L.R.Q., c. C-38 [ci-après L.C.Q.].

11. Ibid., art. 77 L.C.Q. et art. 123.6(2) L.C.Q.

12. Ibid., art. 8 L.C.Q.

13. Ibid., art. 123.13 L.C.Q.

14. M. MARTEL et P. MARTEL, La compagnie au Québec, Montréal, Wilson \& Lafleur, 1993, p. 802. 


\section{2- L'actionnaire et l'endettement de l'entreprise dans le schéma du droit commun}

Au regard des principes du droit commun, il est hautement improbable qu'un actionnaire parvienne à contester avec succès un acte du conseil d'administration dont la conséquence est d'accroître l'endettement de l'entreprise, au simple motif que la décision est viciée par l'incompétence ou l'erreur de jugement, ou encore qu'il s'agit là d'un mauvais calcul économique ou commercial. Si le droit commun place sur l'administrateur de société une obligation de soin, de prudence et de diligence, celle-ci est, somme toute, peu contraignante. En effet, elle se trouve à être fortement relativisée par les profondes réticences des tribunaux nord-américains, y compris québécois, à l'idée de s'immiscer dans la marche des affaires d'une société et de contrôler la légalité d'une décision du conseil d'administration en fonction d'opinions profanes sur sa valeur économique ou commerciale. ${ }^{15}$ La politique judiciaire dominante en Amérique du Nord en est donc une de retenue. En principe, le juge présumera la régularité de la décision des administrateurs; il s'abstiendra de remettre en question le jugement économique ou commercial qui lui est sous-jacent. De même, il se refusera à engager la responsabilité civile personnelle de l'administrateur, même en regard d'une décision qui, de toute évidence, traduit un manque de clairvoyance, voire de compétence. ${ }^{16}$

Dans des circonstances extrêmes, caractérisées par la mauvaise foi de la direction, les conflits d'intérêts ou la négligence outrageusement grossière, il serait sans doute théoriquement possible d'assimiler l'alourdissement de la dette de l'entreprise à un manquement à l'obligation de loyauté de l'administrateur. ${ }^{17}$

15. B. WELling, Corporate Law in Canada - The Governing Principles, 2e éd., Toronto, Butterworths, 1991, pp. 329-336; M. MARTEL et P. MARTEL, ibid., p. 503; J. TURGEON, «Les mesures défensives du conseil d'administration face à une offre publique d'achat», dans R. CRÊTE, Les mesures défensives en matière d'offres publiques d'achat au Canada : aspects juridiques et financiers, Cowansville, Les Éditions Yvon Blais, 1991, p. 155; V. LOUNGNARATH, «Le droit applicable à l'offre publique d'achat au Québec : une entreprise de synthèse», (1994) 35 C. de D. 199, 249.

16. M. MARTEL et P. MARTEL, op. cit., note 14, p. 584.

17. L'obligation de loyauté de l'administrateur est l'équivalent en droit civil québécois des fiduciary duties de la Common Law : voir M. MARTEL et P. MARTEL, op. cit., note 14, p. 506. Voir article 322(2) du Code civil du Québec [ci-après C.c.Q.] qui affirme cette obligation de loyauté. 
Divers recours permettraient alors d'engager la responsabilité civile des administrateurs ou d'obtenir la nullité de l'acte. Dans la même veine, il est à signaler que dans l'affaire ontarienne Re Palmer and Carling O'Keefe Breweries of Canada Ltd., le tribunal a vu dans une fusion, dont l'effet était d'accroître la dette indirectement assumée par les détenteurs d'une catégorie d'actions, un manquement à l'obligation de loyauté de l'administrateur. ${ }^{18}$

\section{3- Le recours statutaire pour oppression de l'actionnaire et l'endettement de l'entreprise}

Le recours pour oppression de l'article 241 L.S.A. représente une piste plus intéressante que le droit commun pour le détenteur de titres désireux d'attaquer une action de la direction ayant pour conséquence d'alourdir le passif de la société. En effet, la décision Re Palmer and Carling O'Keefe Breweries of Canada Ltd. permet d'envisager l'emploi de ce recours statutaire lorsqu'il est établi que l'accroissement de la dette déprécie la valeur des titres du requérant.

En vertu de l'article 241 L.S.A., un actionnaire ou tout autre détenteur de titres peut requérir l'intervention du tribunal lorsqu'une société ou son conseil d'administration accomplit un acte préjudiciable à ses intérêts ou abusif à son égard. Le schéma du recours de l'article 241 L.S.A., maladroitement désigné recours pour oppression de l'actionnaire, donne au juge une vaste discrétion dans l'élaboration des voies de redressement; il peut notamment condamner personnellement les dirigeants à réparer le préjudice subi, ou encore soulever le voile corporatif et atteindre l'actionnaire exerçant le contrôle. L'article 241 L.S.A. est plus qu'un mécanisme procédural, car au fil de ses applications jurisprudentielles, il s'est érigé autour de ce recours un régime de droit substantiel autonome par rapport au droit commun, même s'il en emprunte plusieurs éléments. Le recours pour oppression de l'actionnaire est centré sur le préjudice subi par l'actionnaire et, suivant cette logique, il peut contrer des actes ou des omissions qui ne sont pas empreints de mauvaise foi ou qui se localisent à l'extérieur du domaine des manquements à l'obligation de loyauté de l'administrateur.

18. Re Palmer and Carling O'Keefe Breweries of Canada Ltd, (1989) 67 O.R. (2d) 161, 172 (H.C.). 
La toile de fond de l'affaire Re Palmer and Carling O'Keefe Breweries of Canada Ltd. est une prise de contrôle financée par endettement, suivie d'une opération de fusion. Le tribunal s'est fondé sur le recours statutaire pour oppression de l'actionnaire ${ }^{19}$ afin de sanctionner une opération dont l'effet était d'accroître l'endettement assumé indirectement par un groupe de porteurs de titres. La société I.X.L., filiale à part entière de la société australienne Elders, fit l'acquisition, par la voie d'une offre publique d'achat, de la totalité des actions ordinaires de la société Carling O'Keefe Limited (COL). Cette dernière détenait à part entière la société Carling O'Keefe Breweries (COB). Après cette acquisition, COL proposa de modifier ses statuts constitutifs de manière à racheter les actions privilégiées à un prix au-dessus de la valeur marchande du titre, mais inférieur au prix de rachat existant. La proposition fut battue. On revint à la charge avec un projet de fusion verticale simplifiée entre COL, I.X.L. et COB. La fusion entraînait l'échange des actions privilégiées de COL contre des actions privilégiées d'une nouvelle société, issue de la fusion. Un groupe de détenteurs d'actions privilégiées contesta cette fusion au motif qu'ils se trouvaient à assumer une dette supplémentaire de 400 millions de dollars, sans que ne leur soit versée une compensation quelconque en retour. Les requérants alléguaient que l'opération de fusion leur conférait des titres de moindre valeur, d'où le préjudice. Le tribunal accueillit le recours statutaire pour oppression des détenteurs d'actions privilégiées et ordonna à la société issue de la fusion de racheter les actions privilégiées au prix de rachat initialement prévu dans les statuts constitutifs.

Enfin, le professeur Ziegel s'appuie sur «a small but growing number of cases ${ }^{20}$ pour avancer la thèse que le créancier ordinaire d'une société de régime fédéral peut se prévaloir du recours pour oppression de l'article 241 L.S.A. en regard d'une action dont l'effet est de réduire la valeur ou la qualité de sa créance. En théorie, cette configuration de l'article 241 L.S.A. munit le créancier ordinaire d'un instrument pour combattre un état extrême de sous-capitalisation ou une utilisation outrancière de l'effet de levier. En effet, la sous-capitalisation et l'effet de levier augmentent le risque financier d'une

19. Le recours statutaire pour oppression de l'actionnaire était intenté, dans cette affaire, en vertu de l'article 247 du Ontario Business Corporation Act, S.O. 1982, c. 4; ce recours est un clone du recours de l'article 241 L.S.A.

20. J.S. ZIEGEL, «Creditors as Coporate Stateholders : the Quiet Revolution - an Anglo-Canadian Perspective», (1993) 43 University of Toronto Law Journal 511, 512. 
entreprise et diminuent ainsi la valeur ou la qualité des droits des créanciers ordinaires.

\section{C- La responsabilité civile du prêteur eu égard à la décision d'octroyer un crédit}

En droit français, il est accepté que la responsabilité extra-contractuelle du banquier puisse être engagée en rapport avec l'octroi d'un crédit à une entreprise, et cela, suivant les principes de droit commun. Le tiers au contrat bancaire a ainsi la faculté de poursuivre le banquier qui a consenti un prêt inapproprié, sans s'être préalablement livré à une appréciation de l'utilité et de l'opportunité du crédit. Le banquier est aussi vulnérable au plan de la responsabilité civile professionnelle s'il a contribué à la solvabilité apparente de l'entreprise en octroyant un crédit imprudent. De même, le banquier pourrait, selon certains scénarios, se voir reprocher une faute extra-contractuelle s'il s'engage dans un financement auquel se rapportent des risques interdits, intrinsèquement mauvais ou excessifs. ${ }^{21}$

Au Québec, les tribunaux ne semblent pas convaincus des mérites d'un contrôle de l'octroi du crédit à l'entreprise effectué par le biais du mécanisme de la responsabilité civile extra-contractuelle et, conséquemment, ils se sont abstenus d'emprunter cette voie. Il ne se profile pas non plus de construction doctrinale indigène pouvant servir de support à cette forme d'interventionnisme judiciaire. Les solutions élaborées par la doctrine et la jurisprudence françaises n'ont donc pas été intégrées au droit civil québécois. Il serait étonnant que cette donnée soit modifiée par l'entrée en vigueur du Code civil du Québec.

21. C. GALVADA, «La responsabilité du banquier en cas de faillite de l'entreprise financée», dans La survie des entreprises privées en difficulté, Montréal, Wilson \& Lafleur/ Éditions SOREJ, 1982, pp. 275-287; L. LILKOFF, «La responsabilité du banquier dispensateur de crédit en droit québécois», (1985) 19 R.J.T. 145, 148-150. 


\section{D- Les limites juridiques relatives aux modalités et aux conditions de l'endettement de l'entreprise}

$\mathrm{Au}$ chapitre des modalités et des conditions du financement de l'entreprise, la liberté contractuelle domine. Les principales restrictions à cette liberté touchent l'intérêt exigible, en particulier la divulgation de l'intérêt exigible. Elles se trouvent principalement énoncées dans la Loi concernant l'intérêt, ${ }^{22}$ une loi fédérale qui doit, d'après la Cour suprême du Canada, recevoir une interprétation restrictive. ${ }^{23}$

Les lois et règlements particuliers régissant les diverses catégories d'institutions financières — notamment la Loi sur les banques - comportent aussi des dispositions sur la divulgation de l'intérêt exigible et sa méthode de calcul. $^{24}$

22. L.R.C. 1985, c. I-18. [ci-après L.I.].

23. London Loan and Savings Co. of Canada c. Meagher, [1930] R.C.S. 378, 382; Asconi Building Corp. and Vermette, [1947] R.C.S. 358, 366. Quant à la teneur des restrictions contenues dans la loi, mentionnons que l' article 4 L.I. pose qu' un contrat stipulant un intérêt payable à un taux ou à un pourcentage correspondant à une période inférieure à un an devra énoncer le taux équivalent calculé sur une base annuelle. Autrement, aucun intérêt supérieur au taux ou pourcentage de cinq pour cent n'est exigible. Cette disposition ne s'applique pas à l'hypothèque immobilière, cas traité à l'article 6. En outre, l'article 4 L.I. ne s'applique qu'aux contrats stipulant un taux ou un pourcentage d'intérêt dont la base est une période inférieure à un an; une disposition conventionnelle établissant que l'intérêt est calculé et payable mensuellement ne va pas, à elle seule, placer le contrat en cause dans le champ d'application de l'article 4 L.I. : Bank of Nova Scotia c. Dunphy Leasing Entreprises Limited, [1994] 1 R.C.S. 552, E.A. FISHER, «Advising on Credit and Lending Activities - Current Developments», dans Current Developments in Banking, Toronto, Insight, 1994, pp. 15-16. L'article 6 L.I. vise l'hypothèque immobilière qui garantit un principal ou un intérêt payable par versements périodiques, d'après un système où les versements du principal et de l'intérêt sont confondus ou d'après tout plan ou système comprenant une allocation d'intérêt sur des remboursements stipulés. L'article 6 pose deux grandes exigences. D'abord, l'acte de prêt hypothécaire devra faire mention du capital prêté et du taux d'intérêt exigible à son égard. Ensuite, l'article 6 L.I. prescrit une règle de calcul de l'intérêt : celui-ci devra être calculé annuellement ou semestriellement, mais non d'avance. Voir à ce sujet R. VAILLANCOURT, «Quelques aspects de la Loi sur l'intérêt», (1980) C.P. $d u$ N. 181, 193-199. L'article 8 L.I. concerne les arrérages de créances garanties par hypothèque immobilière. Par cette disposition, les amendes, peines ou intérêts payés en retard sont plafonnés au taux d'intérêt sur le principal non arriéré. Un certain flottement règne quant à la portée réelle de cette disposition : VAILLANCOURT, ibid., 199-201.

24. À titre d'exemple, voir les articles 449 à 454 L.B.; Règlement sur le coût d'emprunt (banques), DORS/92-320, 21 mai 1992. 
Par ailleurs, contrevient à l'article 347 du Code criminel $^{25}$ quiconque conclut une convention ou une entente pour percevoir des intérêts à un taux criminel ou qui perçoit, en totalité ou en partie, des intérêts à un taux criminel. Par taux criminel, il faut entendre un taux d'intérêt effectif de plus de soixante pour cent. $^{26}$

Enfin le Code civil du Québec met en place des mécanismes de contrôle des modalités et conditions de conventions de prêt. L'article 1405 C.c.Q. pose comme principe général que la lésion entre majeurs ne vicie pas le contrat. Le prêt portant sur une somme d'argent fait exception à ce principe général : en effet, l'article 2332 C.c.Q. affirme que le tribunal peut, dans le cas du prêt portant sur une somme d'argent, «prononcer la nullité du contrat, ordonner la réduction des obligations qui en découlent ou, encore, réviser les modalités de leur exécution dans la mesure où il juge, eu égard au risque et à toutes les circonstances, qu'il y a eu lésion à l'égard de l'une des parties».

En outre, s'il s'avère que la convention de prêt est d'adhésion, ${ }^{27}$ l'entreprise endettée sera favorisée dans le processus d'interprétation du contrat. ${ }^{28}$ Dans certaines circonstances, elle pourra obtenir la révision de certaines clauses contractuelles : la clause externe dont l'entreprise endettée ignorait l'existence au moment de la formation de la convention, ${ }^{29}$ la clause illisible ou incompréhensible, ${ }^{30}$ et la clause abusive. ${ }^{31}$

\footnotetext{
25. Code criminel, L.R.C. (1985), c. C-46.

26. Ibid.

27. 1379 C.c.Q.

$28 . \quad 1432$ C.c.Q.

29.1435 C.c.Q.

$30 . \quad 1436$ C.c.Q.

31. 1437 C.c.Q.
} 


\section{II- Le domaine de la notion juridique de dette}

\section{A- Qualification juridique de l'apport externe : dette ou capitaux propres? ${ }^{32}$}

\section{1- Principes généraux}

La qualification de dette ou de capitaux propres revêt une importance particulière en matière d'insolvabilité et de faillite. En effet, les droits du détenteur d'un instrument de capital propre sont impérativement subordonnés à ceux des créanciers en cas de faillite ou de liquidation et il est interdit d'altérer cet ordre de priorité. ${ }^{33}$

Si l'émergence d'instruments financiers hybrides n'a pas ébréché la dichotomie juridique traditionnelle de la dette et des capitaux propres, il reste que cela a singulièrement compliqué le processus de qualification et accru sa subjectivité. Quatre grands principes guident l'exercice de qualification; ils émergent de la jurisprudence et en particulier d'une décision récente de la Cour suprême du Canada, Société d'assurance-dépôt du Canada c. Banque commerciale canadienne. ${ }^{34}$

Premièrement, la qualification suppose une analyse des conventions et des circonstances orientée vers l'objectif d'identifier l'intention commune des parties. $^{35}$

32. Capitaux propres, fonds propres, avoir ou investissement sont considérés comme synonymes; ces expressions correspondent à la terminologie anglaise d' «equity».

33. Sociétéd'assurance-dépôts du Canada c. Banque commerciale canadienne, (1992)7 B.L.R. (2d) 154 (C.S.C.) [ci-après SADC c. BCC], 172; Re Meade, [1951] 1 ch. 774, [1951] 2 All E.R. 168; Laronge Realty Ltd. c. Golconda Investments Ltd., (1986) 7 B.C.L.R. (2d) 90, 63 C.B.R. (N.S.) 76 (C.A.); Halsbury's Laws of England, 4e éd., vol. 3(2), Londres, Butterworths, 1985 p. 315. Ce principe de Common law, fermement établi, est pleinement compatible avec le droit civil québécois et en fait sans doute partie intégrante.

34. Ibid.

35. Ibid., 169, 174-175; La Société coopérative agricole du canton de Granby c. Ministre national du Revenu, (1961) R.C.S. 671. 
Deuxièmement, la substance et les implications économiques de l'instrument financier en cause priment sur sa forme et sa dénomination. ${ }^{36}$

Troisièmement, la réception par le détenteur de l'instrument en cause d'une quote-part des bénéfices milite en faveur de la qualification de capitaux propres, bien qu'une telle caractéristique ne crée pas une présomption absolue que l'on a affaire à un instrument de capitaux propres. ${ }^{37}$ Dans l'affaire Société d'assurance-dépôts du Canada c. Banque commerciale canadienne, la Cour suprême du Canada a établi une distinction entre, d'une part, la quote-part des bénéfices et, d'autre part, l'identification spécifique des bénéfices de l'entreprise comme l'une des sources de remboursement de la somme avancée. Ce dernier facteur ne permet pas, à lui seul, de conclure à la qualification de capitaux propres. ${ }^{38}$ À l'instrument de dette se rattache d'ordinaire une obligation contractuelle de faire des paiements déterminés ou déterminables à des échéances précises; la quote-part des bénéfices, qu'elle prenne ou non la forme de dividendes déclarés et versés, s'oppose conceptuellement à cette obligation.

Quatrièmement, il existe un rapport décisif entre, d'une part, la qualification de dette et, d'autre part, le rattachement à l'instrument financier d'une obligation pour la société de racheter le titre ou d'effectuer un remboursement à une date déterminée ou déterminable. Dans l'affaire Société d'assurance-dépôts du Canada c. Banque commerciale canadienne, la Cour suprême du Canada a indiqué que la qualification de prêt est préférable à celle d'investissement lorsque la convention en cause établit le principe du remboursement des avances consenties à l'entreprise et en précise les modalités. ${ }^{39}$ À l'inverse, une entente qui ne prévoit pas explicitement le remboursement des avances et ses modalités devrait, en toute probabilité, être qualifiée d'investissement ou d'instrument de capitaux propres. ${ }^{40}$

36. D.A. BALL, «Corporate Aspects of Reorganisation and Refinancing», (1984) 2 Nat. Ins. Rev. 4, 5.

$37 . \quad$ Ibid., 4-5.

38. $S A D C$ c. $B C C$, précité, note $33,178$.

39. Ibid., 166 et 181.

40. Ibid., 179; BALL, loc. cit., note 36, 5. 


\section{2- La décision Société d'assurance-dépôts du Canada c. Banque commerciale canadienne}

Eu égard à la problématique abordée dans cette partie, cette décision est significative et mérite une brève présentation. Dans cette affaire, la Cour suprême du Canada avait à qualifier une entente financière élaborée pour venir en aide à la Banque commerciale canadienne. ${ }^{41}$ Cette institution financière éprouvait d'inquiétants problèmes de solvabilité, provoqués par une grave détérioration de son portefeuille de prêts et garanties connexes. Dans le cadre du programme de soutien, le gouvernement fédéral, le gouvernement de l'Alberta, la Société d'assurance-dépôts du Canada et les six plus importantes banques à charte du pays (appelés collectivement les «participants») prirent une participation indivise dans le portefeuille de prêts et garanties connexes de la $\mathrm{BCC}$. La BCC retenait une participation indivise correspondant à la différence entre la valeur nominale comptable du portefeuille (plus de 500 millions de dollars) et l'apport financier des participants. Le programme de soutien était articulé autour d'un certain nombre de conventions, dont les plus importantes étaient l'entente de participation et l'entente de financement par actions.

L'opération était calculée de manière à ce que la part des participants dans chaque élément d'actif du portefeuille de la BCC représente la meilleure appréciation de la portion irrécouvrable de cet élément d'actif. Dans les faits, les participants se trouvaient à avancer à la BCC une somme d'argent correspondant à la valeur nominale comptable de ses prêts douteux. En contrepartie, les participants obtenaient des droits sur des sources de revenu spécifiques. Ainsi, l'entente de participation intéressait les participants aux sommes produites par les éléments d'actifs du portefeuille de la BCC. Il était toutefois convenu que les deniers générés par un élément d'actif devaient d'abord servir la part de la $\mathrm{BCC}$ dans cet élément d'actif. Les participants ne profitaient de cet élément d'actif qu'après le remboursement intégral de la part de la BCC. Aussi, la BCC s'engageait à verser la moitié des revenus avant impôts aux participants. Par ailleurs, l'entente de financement par actions accordait aux participants des bons de souscription qui permettaient d'acquérir à un prix avantageux jusqu'à 75\% des actions ordinaires de la BCC. Les bons de souscription étaient entièrement cessibles.

41. [Ci-après BCC]. 
Les bons de souscription avaient un caractère éventuel. En effet, la mise en oeuvre de l'entente de financement par actions supposait, notamment, une augmentation du capital social autorisé de la BCC, ce qui nécessitait l'approbation des actionnaires et l'autorisation du gouvernement fédéral. Une alternative était donc prévue : les participants obtiendraient des droits sur la totalité des revenus avant impôts si la BCC ne parvenait pas à obtenir avant une date déterminée les autorisations requises. Suivant ce scénario alternatif, la BCC s'engageait, exceptionnellement, à verser des intérêts sur les contributions financières des participants.

Si le feu vert était donné à l'augmentation du capital social autorisé, les sommes obtenues par un participant à même les éléments d'actif ou les revenus avant impôts de la BCC ne pouvaient, cumulativement, excéder le montant de sa contribution financière. Dans le cas contraire, le plafond était fixé par la contribution financière majorée des intérêts. Si ces deux sources s'avéraient insuffisantes pour récupérer un montant égal à la contribution financière ou, dans le scénario alternatif, à la contribution financière majorée des intérêts, la BCC avait l'obligation d'indemniser le participant pour la perte subie. Il est à noter que l'obligation d'indemnisation n'était pas inscrite au bilan comme un élément de passif. Ce traitement comptable était conforme aux directives explicites du Bureau de l'inspecteur général des banques ${ }^{4 l a}$. Aussi, l'entente de participation prévoyait qu'en cas d'insolvabilité ou de mise en liquidation, tout montant non payé constituerait une dette de la BCC. Les participants se voyaient alors attribuer un rang égal à celui des créanciers ordinaires de la BCC.

Malgré toute son ingéniosité, le programme de soutien ne put empêcher la déroute de la BCC.

Dans une opinion unanime rédigée par le juge Iacobucci, la Cour suprême qualifie de prêt la contribution financière de 255 millions. Cette qualification implique que les participants au programme de soutien ont égalité de rang avec les créanciers non garantis de la BCC. Si le plus haut tribunal du pays reconnaît que l'entente financière en cause tient à la fois de l'investissement et du prêt, il opine que ce dernier aspect domine. De l'avis du

41.a Le Bureau de l'inspecteur général des banques est le prédécesseur du Bureau du surintendant des institutions financières. 
juge Iacobucci, la formulation et la substance de certaines dispositions contractuelles reflètent l'intention des parties de constituer une relation de créancier à débiteur. En particulier, le magistrat est influencé par les clauses concernant la récupération des contributions financières par les participants. Les vues du juge Iacobucci sont ainsi synthétisées ${ }^{42}$ :

«En résumé, les termes choisis par les parties dans leurs ententes appuient manifestement la conclusion de la Cour d'appel que le programme d'aide comportait essentiellement un prêt de 255 millions de dollars et non un investissement. Les circonstances qui ont entouré l'opération viennent renforcer cette conclusion plutôt que la miner. Bien que l'opération ait comporté un aspect de financement par actions (les bons de souscription), cet aspect ne suffit pas à lui seul, dans les circonstances, à transformer en un investissement une avance qui constitue essentiellement un prêt. En d'autres termes, bien que l'opération possède certaines "caractéristiques propres aux investissements", celles-ci sont accessoires à la dette constatée par l'arrangement et ne modifient pas le fond de la relation débiteur-créancier créée par les parties relativement à l'avance de 255 millions de dollars consentie à la BCC par les participants. De plus, le fait que le revenu avant impôts de la BCC constituait la principale source de remboursement de l'avance ne modifie en rien cette qualification puisque le remboursement qui devait provenir de cette source était limité à l'avance consentie à la $\mathrm{BCC}$, plus des intérêts éventuels calculés selon le taux préférentiel.»

\section{B- Le traitement comptable et le processus de qualification}

Même si les recommandations du Conseil des normes comptables de l'Institut canadien des comptables agréés ${ }^{43}$ concernant la comptabilité et la vérification n'ont pas, à strictement parler, force de loi, elles représentent la principale référence de la profession comptable et la base des principes comptables généralement reconnus au Canada. Sur la qualification de l'apport financier, les recommandations apportent très peu d'éclairage. Certes, «passif», ${ }^{44}$

42. $S A D C$ c. $B C C$, précité, note $33,169$.

43. [Ci-après I.C.C.A.].

44. Manuel de l'Institut canadien des comptables agréés, paragraphe 1000-32. 
«capitaux propres» ${ }^{45}$ et «prêt» ${ }^{46}$ y sont définis; mais les termes employés ne font qu'exprimer l'intuition commune et des généralités, somme toute, assez banales. Surtout, ces définitions ne contiennent pas de paramètres précis pour la qualification comptable des cas intermédiaires et des instruments hybrides. ${ }^{47}$

L'incidence du traitement comptable sur la qualification juridique d'une contribution financière reste limitée. Dans l'affaire $S A D C$ c. $B C C$, le juge Iacobucci exprime l'opinion qu'en l'occurrence, «le traitement comptable ne revêt pas en soi une grande importance dans la qualification de l'avance». ${ }^{48}$ En conséquence, il rejette l'argument suivant lequel la qualification d'investissement découlerait de la décision comptable de ne pas inscrire comme élément du passif l'obligation d'indemniser les participants à l'entente financière en cause. Le magistrat se garde toutefois de poser une règle générale excluant dans l'absolu l'utilisation du traitement comptable pour déterminer la nature juridique d'une opération. ${ }^{49}$

\section{C- $\quad$ La thèse de l'«equitable investment» et l'article 139 de la Loi sur la faillite et l'insolvabilités ${ }^{50}$}

Suivant la thèse de l' «equitable investment», une opération se présentant comme un prêt à une entreprise, et techniquement structurée comme tel, sera néanmoins reléguée au rang des capitaux propres s'il s'avère que le rendement

45. Ibid., paragraphe 1000.35.

46. Ibid., paragraphe 3025.02.

47. Signalons, toutefois, un projet de recommandation sur les instruments financiers parrainé conjointement par le Conseil des normes comptables de l'Institut canadien des comptables agréés et le Comité international de normalisation de la comptabilité qui aborde dans le détail la problématique de la qualification. Le projet est encore à l'étude et n'est pas encore entré en vigueur. Il y est notamment réitéré que «[c]'est la substance ou réalité économique de l'instrument financier, et non sa forme qui détermine son classement dans le bilan de l'émetteur»: «Instruments financiers, exposé-sondage», CA Magazine, avril 1994, paragraphe 0.053, p. ES12. Par ailleurs, il y est recommandé de classer dans le passif l'action privilégiée assortie d'une obligation de rachat à la charge de la société émettrice : ibid., aux paragraphes 0.053 et 0.054 . C'est là une perspective comptable; d'un point de vue juridique, il apparaît peu vraisemblable que l'action privilégiée à laquelle se rattache une obligation de rachat à une date déterminée ou déterminable soit qualifiée d'instrument de dette plutôt que d'instrument de capital propre.

48. $\quad S A D C$ c. $B C C$, précité, note $33,180$.

49. $\quad S A D C$ c. $B C C$, précité, note $33,181$.

50. L.R.C. (1985), c. B-3 [ci-après L.F.I.]. 
sur le prêt est lié aux bénéfices de l'entreprise. ${ }^{51}$ Cette thèse, issue de la Common Law, a inspiré l' article 139 L.F.I. ${ }^{52}$ Ce dernier et son principe ont cependant une portée limitée. En effet, une distinction s'impose entre la réception par le prêteur d'une quote-part des bénéfices de l'entreprise, d'une part, et l'arrangement qui identifie les bénéfices de l'entreprise comme une source pour le remboursement d'un montant fixe, d'autre part. De la décision $S A D C$. c. $B C C$, il ressort clairement que ce dernier cas échappe à l'application de l'article 139 L.F.I. et de son principe. ${ }^{53}$

\section{D- Les quasi-fonds propres}

Les quasi-fonds propres sont ces dettes subordonnées à l'ensemble de la dette ordinaire. En cas de concours, elles passeront après les créances ordinaires et les titres de participation, d'où l'appellation quasi-fonds propres. ${ }^{54}$ En droit québécois, les quasi-fonds propres ne jouissent pas d'un statut juridique particulier, du moins en ce qui concerne les sociétés industrielles et commerciales. ${ }^{55}$ La subordination de la dette est réalisée par le mécanisme conventionnel : en fait, c'est la technique de la stipulation pour autrui qui est employée dans la plupart des cas. ${ }^{56}$

51. B. CRAWFORD, C.L. CAMPBELL, «The Use of Participation Agreements in Bank Rescues : Bovill's Act and Equitable Subordination in Hybrid Investments», (1993-1994) 9 B.F.L.R. 45, 51; l'expression «equitable investment» est une trouvaille de Crawford et Campbell. L'expression anglaise est conservée dans le texte car à notre avis, la traduction ne rendrait pas tout à fait justice au concept.

52. La jurisprudence ne nous permet cependant pas d'affirmer que cette thèse a acquis la consistance d'un principe de droit positif autonome par rapport au droit statutaire et applicable en l'absence d'un fondement statutaire. Cette remarque vaut autant pour le droit civil québécois que pour la Common law canadienne.

53. $S A D C$ c. BCC, précité, note 33, 188. B. CRAWFORD et C.L. CAMPBELL, loc. cit., note 51, 53-54.

54. G.C. BLAYLOCK, «Leverage : A Corporate Banker's Perspective» (1990), 5 B.F.L.R. 348, 351; L. PAYETTE, «Des priorités et des hypothèques», dans La réforme du Code civil, tome 3, Sainte-Foy, Les Presses de l'Université Laval, 1993, p. 48.

55. Pour certains types de sociétés oeuvrant dans le secteur financier, la loi reconnaît un certain statut aux quasi-fonds propres. C'est le cas des sociétés d'assurance, des sociétés de fiducie et des sociétés d'épargne de régime québécois : voir à titre d'exemple l'article 62.1 de la Loi sur les assurances, L.R.Q., c. A-32 et l'article 193 de la Loi sur les sociétés de fiducie et les sociétés d'épargne, L.R.Q., c. S-29.01.

56. L. PAYETTE, loc. cit., note 54, 48-49, qui s'exprime comme suit sur le sujet : «L'emprunteur stipule dans le contrat d'emprunt ou dans les instruments de dette qu'il émet que le créancier renonce, ou s'oblige à renoncer, au bénéfice du rang égalitaire que lui 


\section{III- L'endettement d'un groupe de sociétés}

\section{A- L'hégémonie de la doctrine du voile corporatif}

La doctrine du voile corporatif, centrée autour du principe énoncé dans le célèbre arrêt britannique Salomon c. Salomon \& Company, ${ }^{57}$ s' affirme comme une valeur solidement ancrée et incontournable du droit des sociétés au Québec. ${ }^{58}$ Selon cette doctrine, la société par actions possède sa propre personnalité juridique. Elle est une entité juridique distincte de ses actionnaires. Formulée d'une autre façon, il y a un voile entre la société et ses actionnaires. Celui-ci n'est soulevé que dans des circonstances exceptionnelles, telle, par exemple, lorsqu'il est établi que la structure corporative sert des fins frauduleuses ou est employée pour contourner la loi. C'est pourquoi une société par actions ne répond pas, en principe, des dettes de ses filiales ou des personnes morales auxquelles elle est liée. Cela vaut même si la sous-capitalisation de la société est manifeste et qu'elle s'accompagne d'opérations destinées, selon toutes apparences, à brimer les créanciers. ${ }^{59}$ Comme le souligne le professeur Ziegel, «inadequate capitalization has rarely, if ever been, a reason in Canada for the Courts' willingness to lift the veil». ${ }^{60}$ Autrement posé, sous-capitaliser une société n'est pas un délit ou un quasi-délit, et n'engage pas la responsabilité extra-contractuelle des actionnaires. C'est la règle en Common Law

procure son droit de concours en vertu du gage commun; ce créancier ordinaire se subordonne ainsi aux autres créanciers ordinaires, qui sont les bénéficiaires de la stipulation. Comme ces arrangements reposent sur le contrat, ils ne sont véritablement scellés que si le promettant (dans le cas discuté il s'agit du créancier) accepte de s'obliger de la manière que le stipulant (l'emprunteur) a prévue et, puisque la stipulation est révocable jusqu'à ce que l'acceptation par le tiers bénéficiaire soit portée à la connaissance du stipulant ou du promettant (art. 1446 C.c.), qu'elle soit portée à la connaissance de l'un ou l'autre de ceux-ci» (notes omises).

57. [1897] A.C. 22.

58. La doctrine du voile corporatif s'est intégrée au droit civil québécois, comme en témoignent d'ailleurs les articles 309 C.c.Q. et 317 C.c.Q.

59. Clarkson Co. c. Zhelka, (1967) 64 D.L.R. (2d) 457 (ont. H.C.); N.C. SARGENT, «Corporate Groups and the Corporate Veil in Canada : A Penetrating Look at Parent-Subsidiary Relations in the Modern Corporate Enterprise», (1988) 17 Manitoba Law Journal 156, 177-178.

60. J.S. ZIEGEL, loc. cit., note 20, 514. 
anglo-canadienne ${ }^{61}$ il n'y a aucune raison de croire que la position des civilistes québécois soit différente.

Au chapitre de l'endettement d'un groupe de sociétés, les tribunaux adoptent donc une attitude conservatrice. Ils se sont ainsi gardés d'endosser, ne serait-ce que partiellement, la théorie dite de l'«entité d'entreprise», ${ }^{62}$ prônée par des commentateurs comme Sargent, ${ }^{63}$ au Canada, et Berle, aux États-Unis. ${ }^{64}$ Cette théorie s'attarde à la réalité économique du groupe de sociétés. Elle impose une responsabilité collective quant aux dettes de chacune d'entre elles, s'il appert que les activités économiques des sociétés sont étroitement liées et forment un tout.

Enfin, il est à signaler que le recours pour oppression de l'article 241 L.S.A. permet de contourner la doctrine du voile corporatif, puisqu'il envisage le redressement d'une situation abusive ou préjudiciable provoquée par l'une des personnes morales du groupe d'une société de régime fédéral ou le conseil d'administration de cette personne morale. Le détenteur de titres de créance d'une société de régime fédéral peut ainsi intenter un recours direct contre une personne morale du groupe de la société dont il détient des valeurs mobilières. Il devra toutefois démontrer que le préjudice ou l'abus subi a pour source la personne morale poursuivie. Le créancier dont les droits ne se rattachent pas à des valeurs mobilières est dans une position plus ambiguë : la définition de plaignant qui se rattache à 241 L.S.A. ne l'inclut pas, mais des voix dans la doctrine et quelques décisions judiciaires ${ }^{65}$ lui reconnaissent l'intérêt requis pour intenter le recours pour oppression.

61. B. WELLING, op. cit., note 15, p. 146.

62. Rural Municipality of Assiniboia c. Suburban Rapid Transit Co., [1931] 2 D.L.R. 862 (C.A. Man.); Clarkson Co. c. Zhelka, précité, note 59.

63. Loc. cit., note 59.

64. Pour Berle, la société par actions doit être analysée comme «an entreprise bounded by economics, rather than as an artificial mystic personality bounded by forms of words in charter, minute books, and books of account» : A.A. BERLE, Jr., «The Theory of Enterprise Entity», (1947) 47 Colum. L. Rev. 343, cité par N.C. SARGENT, loc. cit., note 59, 156.

65. J.S.ZIEGEL, loc. cit., note 20, 512, 527-529; T.M. DOLAN, «Piercing the Corporate Veil», (1990) 5 National Creditor/Debtor Review, 14-15, de même que la jurisprudence citée par ces commentateurs. 


\section{B- La «subordination reconnue en equity»}

Cette doctrine, américaine ${ }^{66}$ dans son style et ses racines, procède de la compétence d' «equity» des tribunaux. Elle autorise le juge à rétrograder des réclamations faites validement à l'égard de l'actif d'une personne insolvable, lorsqu'elles découlent d'une conduite inéquitable et préjudiciable aux intérêts des autres créanciers. ${ }^{67}$ Aux États-Unis, la doctrine est notamment applicable à la créance d'une société sur sa filiale insolvable dans le cas où cette dernière souffre d'une sous-capitalisation aiguë. Aussi, on pourra la faire intervenir s'il est établi que la société domine entièrement les affaires de sa filiale et qu'elle s'est servie de sa position de contrôle pour appauvrir sa filiale ou lui porter préjudice. ${ }^{68}$ Du côté canadien, la doctrine de la «subordination reconnue en equity» évolue dans une zone grise. Ainsi, dans l'affaire $S A D C$ c. $B C C$, un argument basé sur cette doctrine fut plaidé. Le juge Iacobucci, qui rédigea les motifs de la Cour suprême, a cependant organisé son raisonnement de manière à ne pas avoir à décider si cette doctrine fait partie du droit positif canadien. La jurisprudence des tribunaux inférieurs est quant à elle peu consistante et ambiguë. ${ }^{69}$

$\mathrm{Au}$ Québec, la doctrine de la «subordination reconnue en equity» soulève, en plus, le problème particulier de son intégration au droit civil québécois. L' «equity» représente, en effet, un agrégat juridique étranger à la tradition du droit civil québécois, qui a une fonction supplétive en matière de faillite. $^{70}$

66. Pepper c. Litton, 308 US 295 (US Va, 1939); Taylor c. Standard \& Gas and Electric Co., 306 US 307 (1939).

67. L.J. CROZIER, «Equitable subordination of claims in Canadian Bankruptcy Law», 7 C.B.R. (3d) 40, 41-42.

68. N.C. SARGENT, loc. cit., note 59, 180; L.J. CROZIER, ibid., 41.

69. Crozier considère que la décision Laronge Realty Ltd. c. Golconda Investments Ltd., (1986) 63 C.B.R. (N.S.) 74, 7 B.C.L.R. (2d) 90 (C.A.) est favorable à la doctrine : L.J. CROZIER, ibid., p. 41. D'autres décisions judiciaires récusent la doctrine de la «subordination reconnue en equity»: Aevo Co. c. D \& A MacLeod Co., (1991) 4 O.R. (3d) 368 (Ont. Gen. Div.); Re Orzy, (1923) 3 C.B.R. 737, [1924] 1 D.L.R. 250 (Ont. C.A.); voir aussi Olympia \& York Developments Ltd. c. Royal Trust Co., (1993) 8 B.L.R. (2d) 69 où la décision Aevo Co. c. $D \& A$ MacLeod Co., ibid., est citée en obiter.

70. L'article 183(1)(b) L.F.I. donne à la Cour supérieure du Québec une «compétence en droit et en équité». Le domaine de la compétence en équité (equity) est matière à controverse. Deux écoles de pensée s'affrontent : la première prône l'application dans une procédure de 


\section{IV- Les sûretés générales conventionnelles}

Les sûretés générales portant sur des universalités de biens d'entreprise découlent essentiellement de deux instruments juridiques : le Code civil du Québec et la Loi sur les banques.

\section{A- Le Code civil du Québec et les sûretés générales conventionnelles}

Le Code civil du Québec, entré en vigueur le premier janvier 1994, porte une réforme majeure du droit des sûretés. Le nouveau cadre est marqué par la préoccupation du législateur d'unifier le système des sûretés et de renforcer sa cohérence interne. Ainsi, des institutions de l' «ancien droit» comme le privilège du constructeur, ${ }^{71}$ le transport des créances en garantie, ${ }^{72}$ les sûretés octroyées en vertu de la Loi sur les pouvoirs spéciaux des corporations ${ }^{73}$ ou de la Loi sur les connaissements, les reçus et les cessions de biens en stock ${ }^{74}$ le nantissement commercial ${ }^{75}$ ou le gage ${ }^{76}$ sont remplacées par un concept unique d' hypothèque. Pivot du nouveau régime des sûretés, l'hypothèque du Code civil du Québec se distingue par sa grande souplesse au titre de l'assiette des biens donnés en garantie. La nouvelle hypothèque peut ainsi grever un bien particulier ou une universalité de biens; l'objet de l'hypothèque est corporel ou incorporel, meuble ou immeuble. Il est par ailleurs manifeste que, par sa réforme, le législateur poursuivait l'objectif de faciliter l'obtention de crédit pour l'entreprise. Renforcement de la cohérence des règles, souplesse dans la définition de l'objet de la garantie, et soutien au financement de l'entreprise, sont donc trois grandes idées qui animent la nouvelle hypothèque.

faillite de l'ensemble des droits et des institutions provenant de «l'equity»; la seconde limiterait le champ d'application de l'«equity» à l'interprétation des dispositions de la Loi sur la faillite et l'insolvabilité et de ses procédures. Voir A. BOHÉMIER, Faillite et insolvabilité, tome 1, Montréal, Thémis, 1992, p. 579, où l'auteur synthétise élégamment la controverse et prend parti pour la seconde école de pensée.

71. Articles 2009 à $2013 \mathrm{f}$ du Code civil du Bas-Canada [ci-après C.c.B.C.].

72. 1571d) C.c.B.C.

73. L.R.Q., c. P-16 (modifiée par la Loi sur l'application de la réforme du Code civil, L.Q. (1992), c. 57) ci-après L.P.S.C...

74. L.R.Q., c. C-53 (abrogée par la Loi sur l'application de la réforme du Code civil, ibid., article 520).

75. 1979e) à 1979k) C.c.B.C.

76. 1968 à 1979 C.c.B.C. 
Au chapitre des sûretés générales, le Code civil du Québec crée deux grandes institutions : d'une part, l'hypothèque sans dépossession sur une universalité de biens meubles, qui est la principale forme d'hypothèque universelle, ${ }^{77}$ et d'autre part, l'hypothèque ouverte. Ces deux formes d'hypothèques jouent un rôle majeur dans le système mis en place pour succéder aux sûretés de la Loi sur les pouvoirs spéciaux des corporations, une législation de 1914 qui prévoyait des sûretés spéciales pour garantir le paiement de titres d'emprunt émis par les sociétés et, en règle générale, répartis chez une pluralité d'investisseurs. Dans sa démarche, le législateur québécois s'inspirait fortement de la «floating charge» de la Common Law. Le mécanisme élaboré dans la L.P.S.C. s'articulait autour d'un acte de fiducie et confiait un rôle important au fidéicommissaire, chargé «d'administrer» les sûretés octroyées et de représenter les intérêts des détenteurs d'obligations vis-à-vis le débiteur. Tout cela a été aboli avec l'entrée en vigueur du Code civil du Québec. C'est désormais l'hypothèque en général, et les formes d'hypothèques ci-haut mentionnées en particulier, qui remplissent la fonction des sûretés spéciales de la L.P.S.C. Le Code civil du Québec reconnaît d'ailleurs explicitement à son article 2692 C.c.Q. que l'hypothèque peut être utilisée pour garantir le paiement d'obligations, ou d'autres titres d'emprunt, à condition qu'elle soit constituée «par acte notarié en minute, en faveur du fondé de pouvoir des créanciers.»

L'hypothèque sur une universalité de biens, ou hypothèque universelle, peut seulement être consentie par une personne ou un fiduciaire qui exploite une entreprise. ${ }^{78}$ Dans le système du Code civil du Québec, l'entreprise est définie libéralement comme «une activité économique organisée, qu'elle soit ou non à caractère commercial, consistant dans la production ou la réalisation de biens, leur administration ou leur aliénation, ou dans la prestation de services». ${ }^{79}$ L'universalité peut englober des biens futurs, tout comme il est théoriquement possible qu' elle regroupe des biens immeubles. L'article 2684 C.c.Q. fournit des exemples d'universalités mobilières, parmi lesquels on retrouve l'outillage, le matériel d'équipement professionnel, les créances et comptes-clients, les inventaires de matières premières, de produits finis et de produits en cours de transformation. Concept souple, l'universalité connaît néanmoins certaines

\footnotetext{
77. 2666 C.c.Q.

78. 2684 C.c.Q.; voir cependant 27 L.P.S.C. et 34 L.P.S.C. qui reconnaissent cette faculté à une personne morale n'exploitant pas une entreprise.

79 1525 C.c.Q.
} 
limites : en effet, il se déduit de l'article 2697 C.C.Q que dans le cas d'une hypothèque sans dépossession sur une universalité de biens meubles, les biens hypothéqués doivent avoir une nature commune pour que l'on puisse les inclure dans la même universalité.

De l'énumération non-limitative de l'article 2684 C.c.Q., il ressort qu'une entreprise a la faculté de donner en garantie l'ensemble de ses actifs, par la combinaison judicieuse d'hypothèques immobilières et mobilières sur des biens particuliers avec des hypothèques sur une universalité de biens meubles.

La plupart du temps, il est impératif que l'entreprise conserve la possession physique et juridique des biens meubles hypothéqués pour fonctionner correctement. D'où le mécanisme de l'hypothèque mobilière sans dépossession prévu par le Code civil du Québec qui permet à l'exploitant d'une entreprise $^{80}$ de rester en possession des biens meubles hypothéqués. Le bien meuble spécifique et l'universalité mobilière peuvent faire l'objet d'une hypothèque mobilière sans dépossession.

L'hypothèque mobilière universelle, tout comme d'ailleurs l'hypothèque mobilière spécifique, «n'emporte pas de droit de suite sur le bien meuble vendu dans le cours des activités de l'entreprise et s'éteint à l'égard de ce bien». ${ }^{81} \mathrm{C}$ 'est là un cas d'exception. Plutôt, l'hypothèque se reporte sur le bien acquis en remplacement. S'il n'y a pas de remplacement du bien aliéné, le report se fait «sur les sommes d'argent provenant de l'aliénation, pourvu que celles-ci puissent être identifiées». ${ }^{82}$

Élaborée pour le bénéfice exclusif de la personne ou du fiduciaire qui exploite une entreprise,$^{83}$ l'hypothèque ouverte affectera, suivant la volonté des parties, non seulement des biens spécifiques, mais aussi des universalités de biens, des combinaisons de biens spécifiques et d'universalités, ou encore, l'ensemble des biens mobiliers et immobiliers de l'entreprise. Par ailleurs, elle peut englober des biens futurs. Ces particularités en font un mécanisme de sûreté générale. Le propre de cette sûreté est de suspendre les principaux effets

80. 2683 C.c.Q

81. L. PAYETTE, loc. cit., note 54, 125; 2674 C.c.Q.; 2700 C.c.Q.

82. 2674 C.c.Q.; ce mécanisme de report est aussi exceptionnel.

83. 2686 C.c.Q.; voir cependant 27 L.P.S.C. et 34 L.P.S.C. 
hypothécaires jusqu'au moment où, dans la foulée d'un défaut sur l'obligation principale, le créancier provoque la clôture de l'hypothèque au moyen d'un avis dénonçant le défaut et la clôture de l'hypothèque. L'hypothèque ouverte donne une protection limitée au créancier car c'est l'inscription de l'avis de clôture et non pas la publication de l'hypothèque au moment de sa constitution qui détermine le rang. ${ }^{84} \mathrm{C}$ 'est aussi cet événement qui fixe l'assiette de biens sur lesquels le recours hypothécaire s'exercera ${ }^{85}$ : en effet, l'hypothèque ouverte ne devient opposable aux tiers que par l'inscription de l'avis de clôture, ${ }^{86}$ de sorte qu'avant ce moment, le créancier ne dispose pas d'un droit de suite sur les biens affectés à la garantie. Toutefois, il faut signaler que le détenteur d'une hypothèque ouverte aura préséance sur le créancier ayant inscrit son hypothèque après que la sienne ait été inscrite, si le recours hypothécaire exercé par le détenteur de l'hypothèque ouverte est celui de la prise de possession des biens à des fins d'administration. ${ }^{87}$

L'hypothèque ouverte prend rang et effet avec l'inscription de l'avis de clôture. C'est ce qui la distingue fondamentalement de l'hypothèque mobilière sans dépossession sur une universalité. Le rang de ce dernier type d'hypothèque est déterminé par la publication de l'hypothèque au moment de sa constitution et c'est à partir de cet événement que la sûreté devient opposable aux tiers. L'hypothèque mobilière sans dépossession sur une universalité est donc une sûreté générale plus forte que l'hypothèque ouverte.

Mentionnons, en finale et en périphérie, que l'entreprise se singularise non seulement en regard de l'accessibilité à certaines formes d'hypothèques, mais aussi au plan des recours. Le Code civil du Québec prévoit quatre types de recours hypothécaires en cas de défaut du débiteur : la vente sous contrôle de justice, la prise en paiement, la vente par le créancier et la prise de possession à des fins d'administration. Or les deux derniers types de recours sont au bénéfice exclusif du créancier qui détient une hypothèque sur les biens d'une entreprise.

$\begin{array}{ll}84 . & 2955 \text { C.c.Q. } \\ 85 . & 2719 \text { C.c.Q. } \\ 86 . & 2716 \text { C.c.Q. } \\ 87 . & 2721 \text { C.c.Q. }\end{array}$




\section{B- $\quad$ Les sûretés de la Loi sur les banques}

Vu l'importance du rôle joué par les banques à charte dans la fonction d'intermédiation des capitaux, il n'y a rien d'étonnant à ce que la loi régissant ces institutions - une loi fédérale, la Loi sur les banques — s'impose comme une pièce législative significative, voire incontournable, du droit commercial. Cette loi présente notamment la caractéristique de créer des sûretés particulières, par ses articles 425 à 436. Au Québec, les sûretés bancaires viennent s'ajouter aux sûretés reconnues par le droit civil du Québec qui, par ailleurs, remplit sur le territoire québécois une fonction supplétive et sert de référence conceptuelle. ${ }^{88}$

S'agissant des entreprises industrielles ou commerciales, la sûreté particulière créée par la Loi sur les banques se présente, essentiellement, comme une garantie sur les inventaires du débiteur. ${ }^{89}$ Les banques à charte en sont les bénéficiaires exclusifs. Les biens futurs entrent dans le champ de la sûreté bancaire. Suivant l'article 428(1) L.B., le rang de la sûreté bancaire est déterminé par la date de la convention constituant la garantie, ${ }^{90}$ ce qui signifie, notamment, qu'elle prime toute hypothèque publiée après cette date. Selon certains, la garantie bancaire serait assortie d'un pouvoir de prise de possession s'exerçant sans intervention judiciaire préalable. ${ }^{91}$ La proposition fait cependant l'objet de sérieuses controverses. ${ }^{92}$

Un certain flottement idéologique règne quant à la nature juridique de la sûreté bancaire. Dans L'affaire Banque canadienne nationale c. Lefaivre, ${ }^{93}$ la Cour d'appel du Québec a exprimé l'opinion que la sûreté particulière prévue

88. R.A. MacDONALD, «Security Under Section 178 of the Bank Act : A Civil Law Analysis», (1983) 43 Revue du Barreau 1008, 1016.

89. 426(a) L.B., 426(b) L.B.

90. R. DEMERS, Le financement de l'entreprise - aspects juridiques, Sherbrooke, Les Éditions Revue de droit Université de Sherbrooke, 1985, pp. 170-171 : contra, R.A. MacDONALD, loc. cit., note 88, 1029.

91. R. DEMERS, ibid., 165; M. PAQUET, «Pouvoir de prise de possession informelle dans le cas des garanties des alinéas 178(1) a) et b) de la Loi sur les banques; Existence ou inexistence», [1984] 44 Revue du Barreau 333, 351-356.

92. R.A. MacDONALD, loc. cit., note 88, 1020. La question a été commentée par la Cour suprême du Canada dans l'affaire BNC c. Atomic Slipper Co., [1991] 1 R.C.S. 1059 mais le tribunal s'est gardé de prendre position et d'infirmer les assertions de la Cour d'appel du Québec sur ce point spécifique : ibid., 1074. Le débat n'est donc pas clos.

93. [1951] B.R. 83 (C.A.). 
dans la Loi sur les banques confère un droit de propriété sui generis sur les biens affectés à la garantie. Cette qualification a été vigoureusement et admirablement combattue par le doyen MacDonald. À la suite d'une démonstration éloquente et rigoureuse faite sous l'empire du Code civil du Bas-Canada, celui-ci conclut que le droit inhérent à la sûreté bancaire est un «non-codal, statutory, accessory real right», assimilable à une forme de nantissement. ${ }^{94} \mathrm{Ce}$ débat prend une tournure inédite avec le schéma des sûretés proposé par le Code civil du Québec. Dans son concept et ses caractéristiques, la sûreté bancaire présente, en effet, d'importantes similitudes avec l' hypothèque mobilière sans dépossession. Pour cette raison et parce que le droit civil constitue, au Québec, la référence conceptuelle, il apparaît conséquent et indiqué de modéliser la sûreté bancaire comme une forme d'hypothèque mobilière sans dépossession.

\section{V- Le redressement de l'entreprise excessivement endettée}

\section{A- La réalisation des sûretés et la théorie de l'abus des droits contractuels}

En matière de prêt et de réalisation de sûretés, l'attitude dominante des tribunaux québécois se caractérisait, jusqu'à tout récemment, par une application rigoureuse et zélée du principe de la force obligatoire des contrats. ${ }^{95}$ Ce qui se traduisait, en particulier, par des hésitations à reconnaître des obligations à la charge du créancier qui ne procèdent pas de la lettre du contrat. Le principe de la force obligatoire des contrats s'est cependant vu vigoureusement relativisé dans l'importante affaire Houle c. Banque nationale $d u$ Canada ${ }^{96}$ et ce, par le jeu de l'élargissement de la théorie de l'abus des droits contractuels et de l'application particulière qui en a été faite dans le domaine du droit bancaire. Suite à l'examen de la situation financière de la société débitrice, la banque avait décidé d'exercer ses droits contractuels et de rappeler son prêt

94. R.A. MacDONALD, loc. cit., note 88, 1027; voir aussi la décision de la Cour d'appel du Québec dans Atomic Slipper Co. c. Banque nationale du Canada, [1988] R.J.Q. 2087 (C.A.); s'il est vrai que la Cour Suprême du Canada a renversé la décision de la Cour d'appel du Québec, il reste que sur ce point spécifique, la Cour Suprême du Canada n'a pas récusé explicitement le raisonnement du Juge Malouf; en effet, dans sa résolution du litige, le plus haut tribunal du Canada n'a pas cru nécessaire de se prononcer sur la nature des droits inhérents à la garantie bancaire dans le système civiliste québécois.

95. L. LILKOFF, loc. cit., note 21, 150.

96. [1990] 3 R.C.S. 122. 
à demande. Après seulement une heure de négociations avec les dirigeants de la débitrice, la banque a signifié une mise en demeure et pris immédiatement possession des actifs affectés à la garantie. Le créancier a procédé à la liquidation des actifs en moins de trois heures. Les diverses conventions liant la banque et la société autorisaient la banque à rappeler le prêt sur demande et à réaliser ses sûretés sans préavis. Néanmoins, la Cour suprême du Canada, dans une opinion unanime rédigée par le juge L'Heureux-Dubé, affirme, sur la base de la théorie de l'abus des droits contractuels, que la banque a commis une faute civile. Le tribunal exprime l'avis que l'abus de droit réside, non pas dans le rappel du prêt qui était fondé sur des critères économiques objectifs, mais dans la prise de possession et la liquidation précipitée des actifs de la société. La banque a abusé de ses droits contractuels en ne laissant pas à la débitrice un délai raisonnable pour lui permettre de rembourser le prêt après avoir été mise en demeure. Peu importe que la banque ait agi sans malice ou qu'il y ait eu absence de mauvaise foi : pour mesurer l'abus de droit, la Cour suprême du Canada endosse le critère objectif de l'exercice raisonnable d'un droit. Or, cette grille d'appréciation ne requiert pas une preuve de malice ou d'absence de bonne foi. ${ }^{97}$

Décision marquante, Houle c. Banque nationale du Canada contribuera à établir un meilleur équilibre des droits des parties à une convention de prêt. Elle invite à la civilité dans les rapports entre l'institution prêteuse et une société débitrice qui éprouve des difficultés financières. Par ailleurs, les principes posés dans cette affaire, ceux qui concernent l'abus de droit contractuel et le délai raisonnable en particulier, peuvent, dans certaines circonstances, être des données significatives pour l'entreprise en difficulté qui élabore une stratégie de redressement.

\section{B- La technique de l' «in-substance defeasance»}

«In substance defeasance» fait référence à une technique d'origine américaine par laquelle une société par actions sort une dette de son bilan en plaçant irrévocablement dans une fiducie des actifs financiers et en les affectant exclusivement au remboursement de la dette, en capital et en intérêts. L'apparence du bilan et des ratios financiers y gagne; l'entreprise se trouve donc

97. Ibid., 155 
dans une meilleure posture pour obtenir des crédits supplémentaires, ou recueillir des capitaux frais via un appel public à l'épargne, par exemple. Il est à noter que l'«in-substance defeasance» est d'abord une manipulation comptable. Sur le plan juridique, il y a survivance de la dette. La technique de l'«in-substance defeasance» ne décharge pas la société de ses obligations en vertu de la convention primitive constituant la dette. Le créancier conserve tous ses recours contre la société et peut se fonder sur la convention primitive pour réclamer de la société le remboursement de la dette, si les actifs en fiducie s'avèrent insuffisants. ${ }^{98} \mathrm{~L}$ ' «in-substance defeasance» est à distinguer de la «legal defeasance» qui suppose novation par changement de débiteur et partant, extinction du lien de droit inhérent à la convention primitive entre la société et son créancier.

Le droit civil québécois ne pose pas d'obstacle à une opération d' «in-substance defeasance» : il y reconnaît, fondamentalement, une forme de délégation imparfaite. ${ }^{99}$ En outre, la fiducie du C.c.Q. ${ }^{100}$ se présente comme un réceptacle tout à fait adéquat pour les actifs financiers affectés au service de la dette.

La faisabilité d'une opération d' «in-substance defeasance» se ramène à la question de savoir si les règles comptables autorisent à déplacer hors du bilan, d'une part, une dette qui n'est pas éteinte et demeure, sur le plan juridique, à la charge de la société, et d'autre part, les actifs affectés à son remboursement. À ce chapitre, il règne au Canada un certain flottement normatif, ce qui n'a pas empêché des sociétés aussi sérieuses qu'Air Canada de recourir à cette technique. ${ }^{101}$ Comme le rapporte John A. Zinn, les organismes responsables de l'établissement des normes comptables au Canada n'ont pas jusqu'à maintenant formulé de recommandations formelles sur l' «in-substance defeasance». Cela étant posé, il faut souligner la pertinence d'un projet de recommandation du Conseil des normes comptables de l'I.C.C.A. intitulé «Instruments financiers». Le projet a été initialement présenté en septembre 1991 et a fait l'objet d'une

98. J.A. ZINN, «Advancements in Off-Balance Sheet Financing- Part 1», 13 National Banking Law Review 24, 25.

99. J.-L. BAUDOUIN, Les obligations, $4^{\mathrm{e}}$ édition, Cowansville, Les éditions Yvon Blais Inc., 1993, pp. 547-551.

100. 1260 C.c.Q. à 1298 C.c.Q.

101. J.A. ZINN, loc. cit., note 98,27 et 32 . 
révision en 1993, produite sous forme d'exposé-sondage. Le projet de recommandation prévoit qu'un actif financier ou un passif financier ne peut pas être sorti du bilan si la transaction ne s'accompagne pas d'un transfert vers le tiers de la «quasi-totalité des risques et avantages rattachés à l'actif ou au passif», ${ }^{102}$ ce qui revient à désavouer la technique de l'«in-substance defeasance». Le projet de recommandation n'a pas encore franchi toutes les étapes requises pour l'adoption de normes comptables formelles et intégrées au Manuel de l'I.C.C.A., ce qui a amené un commentateur ${ }^{103}$ à suggérer que l'expérience américaine devait, jusqu'à nouvel ordre, servir de guide normatif en matière d' «in-substance defeasance». En effet, l'information financière doit être rapportée selon les principes comptables généralement reconnus au Canada; pour déterminer leur substance, il est permis de se référer à la pratique étrangère relative à des circonstances similaires, et aux normes adoptées par des organismes reconnus d'autres juridictions. ${ }^{104}$ En 1983, le U.S. Financial Accounting Standard Board (F.A.S.B.), l'organisme chargé d'établir les normes comptables aux États-Unis, publiait un énoncé qui abordait la problématique de l'«in-substance defeasance». En résumé, le document reconnaît que dans le cadre d'une opération d' «in-substance defeasance», une dette peut être sortie du bilan si certaines conditions sont satisfaites. Mentionnons quelques-unes d'entre elles : les actifs financiers sont placés irrévocablement en fiducie; il s'agit d'actifs à faible risque, nommément des titres d'emprunt de l'État, des titres d'emprunt garantis par l'État ou encore, des titres d'emprunt assortis d'une sûreté sur des titres d'emprunt de l'État ou sur des titres d'emprunt garantis par l'État; la probabilité que le débiteur soit tenu d'entrer en scène parce que les actifs placés en fiducie sont insuffisants est minime, sinon insignifiante; enfin, il est requis que la distribution des flux monétaires produits par les actifs financiers en fiducie corresponde à celle des paiements en intérêts et capital sur la dette en cause. ${ }^{105}$

102. Conseil des normes comptables de l'I.C.C.A., «Instruments financiers», C.A. Magazine, avril 1994, ES 1, paragraphe 0.028, loc. cit., note 47.

103. J.A. ZINN, loc. cit., note 98, 26.

104. Ibid., 26.

105. D. GRAY, M.A. SEVILLE, «In-Substance Defeasance : Altering the Shape of Debt Management», dans Journal of Accountancy, janvier 1985, pp. 100-107. 


\section{C- La procédure concordataire de la Loi sur la faillite et l'insolvabilité}

En 1992, le législateur fédéral remaniait en profondeur sa législation sur la faillite. En particulier, la nouvelle Loi sur la faillite et l'insolvabilité $e^{106}$ modifie sensiblement les règles relatives à la proposition et au concordat qui s'adressent à l'entreprise. Le système de la L.F.I. cherche à favoriser la conclusion d'arrangements concordataires. Il tend ainsi vers l'objectif d'accroître les chances de survie des entreprises en difficulté financière.

Le concordat de la L.F.I. est une convention collective et judiciaire ${ }^{107}$. Convention, car il constate une entente entre l'entreprise insolvable et ses créanciers quant au remboursement et l'extinction des créances. Collective, car la proposition faite par le débiteur doit recevoir l'assentiment du collectif des créanciers. Judiciaire, car le régime concordataire ne produira pas d'effet à moins que le tribunal ne ratifie la proposition du débiteur. Contrairement à la faillite, le concordat n'implique pas le dessaisissement de débiteur : celui-ci continue d'administrer son patrimoine. La procédure concordataire de la L.F.I. est organisée autour de quatre éléments : la proposition, l'avis d'intention, l'acceptation des créanciers et la ratification du tribunal.

La proposition se présente comme une offre soumise par le débiteur insolvable à ses créanciers ordinaires et accessoirement, aux créanciers garantis ou à certains d'entre eux. Il résulte de la nature conventionnelle du concordat que le débiteur jouit d'une discrétion étendue dans la formulation de sa proposition. ${ }^{108}$ En particulier, le débiteur peut constituer dans sa proposition des catégories de créanciers ordinaires, et moduler ses offres en fonction de ces catégories. ${ }^{109}$ La créativité du débiteur reste tempérée par quelques contraintes. Par exemple, il est nécessaire que la proposition respecte l'ordre de priorité des créanciers établi par la loi dans la distribution des biens du débiteur et qu'elle prévoie le paiement des honoraires et dépenses du syndic. ${ }^{110}$ Aussi, la proposition doit stipuler le paiement intégral de toutes les sommes dues à la

106. Précitée, note 50.

107. A. BOHÉMIER, Faillite et insolvabilité, tome 1, Montréal, Éditions Thémis, 1992, p. 296.

108. Ibid., p. 298.

109. 50(1.2) L.F.I.

110. 60(1) L.F.I. et 136 L.F.I. 
Couronne à titre de déduction à la source, ${ }^{111}$ le paiement de certains salaires, ${ }^{112}$ de même que le paiement de l'indemnité au locateur. ${ }^{113}$ Innovation significative introduite par la réforme de 1992, le débiteur a la possibilité de s'adresser aux créanciers garantis. Ceux-ci sont regroupés en catégorie selon la similitude de leurs intérêts. La proposition peut être limitée aux créanciers d'une ou de plusieurs catégories mais il est alors impératif que l'offre soit faite à tous les créanciers garantis de chacune des catégories visées. La détermination des catégories et l'entreprise de classification sont soumises au contrôle judiciaire. ${ }^{114}$

La L.F.I. offre au débiteur la possibilité d'enclencher la procédure concordataire par un avis d'intention exprimant la volonté du débiteur de faire une proposition. ${ }^{115}$ Les principaux effets du dépôt de l'avis d'intention sont de suspendre les recours de tous les créanciers, ordinaires comme garantis, et de geler les rapports contractuels. La proposition doit suivre dans les 30 jours du dépôt de l'avis d'intention, bien que le tribunal ait le pouvoir de proroger ce délai. Le dépôt de la proposition produit lui aussi un gel contractuel et un effet suspensif mais seulement à l'égard des créanciers visés par la proposition. C'est dire qu' un créancier garanti appartenant à une catégorie exclue de la proposition sera en mesure de réaliser sa garantie après le dépôt de la proposition.

La loi exige que les créanciers ordinaires, réunis en assemblée, acceptent la proposition du débiteur. Le seuil d'acceptation est fixé à une double majorité, soit la majorité simple en nombre et la majorité des deux tiers en valeur des créanciers non garantis votant sur la proposition. Si des catégories de créanciers ont été créées par la proposition, les créanciers votent par catégorie. La proposition doit alors être acceptée par les majorités requises dans chacune des catégories, selon l'avis exprimé par le professeur Bohémier. ${ }^{116}$

Les créanciers garantis des catégories mentionnées dans la proposition sont aussi appelés à se prononcer sur celle-ci, via un vote par catégorie. Le vote des créanciers garantis est régi par un système de double majorité, identique à

111. A. BOHÉMIER, op. cit., note 107, p. 408.

112. Ibid., pp. 408-409.

113. Ibid., pp. 409-410.

114. $50(1.5)$ L.F.I.

115. 50.4 L.F.I.

116. 54(1)d) L.F.I.; A. BOHÉMIER, op. cit., note 107, pp. 423-424. 
celui qui gouverne le vote des créanciers ordinaires. L'acceptation de la proposition par une catégorie de créanciers garantis lie tous les membres de la catégorie. Ce vote des créanciers garantis n'a cependant pas d'incidence sur l'acceptation de la proposition car «[c]'est la décision des créanciers non garantis qui détermine si la proposition concordataire est acceptée ou non». ${ }^{117}$ Le rejet de la proposition par les créanciers garantis d'une catégorie donnée implique que ceux-ci ne seront pas assujettis au concordat et qu'ils pourront réaliser librement leur garantie.

Pour que le concordat prenne effet, la proposition acceptée par les créanciers doit être ratifiée par le tribunal. Celui-ci va s'assurer que la proposition remplit les exigences de la loi. ${ }^{118} \mathrm{Si}$ c'est le cas, il jouit d'une discrétion étendue pour approuver ou refuser d'approuver la proposition. ${ }^{119} \mathrm{Le}$ contrat prend effet avec la ratification de la proposition, et à partir de ce moment, il gouverne les relations entre le débiteur, les créanciers ordinaires et les créanciers garantis des catégories ayant approuvé la proposition.

Le rejet de la proposition par les créanciers ordinaires ou par le tribunal entraîne la faillite du débiteur. Par ailleurs, le défaut du débiteur de se conformer à la proposition est une cause d'annulation du concordat. ${ }^{120}$ L'annulation du concordat entraîne la faillite automatique du débiteur. ${ }^{121}$

\section{D- Le redressement de l'entreprise à l'extérieur de la faillite : la Loi sur les arrangements avec les créanciers des compagnies}

En adoptant, en 1932, la Loi sur les arrangements avec les créanciers des compagnies ${ }^{122}$ le législateur fédéral cherchait à aménager un cadre juridique extérieur à celui de la faillite, dans lequel les entreprises foudroyées par la «Grande Dépression» pourraient négocier des compromis avec leurs créanciers, et ainsi éviter la faillite. Depuis cette époque, la mission sociale de cette loi n'a jamais été reniée et conséquemment, les tribunaux n'ont pas eu de réticence à

117. A. BOHÉMIER, op. cit., note 107, p. 428; 54(2) L.F.I.

118. 60(1) L.F.I. et 136 L.F.I.; op. cit., note 107, pp. 408-410.

119. 60(5) L.F.I.; 59 L.F.I.

120. A. BOHÉMIER, op. cit., note 107, pp. 437-438; 63 L.F.I.

121. A. BOHÉMIER, op. cit., note 107, p. 439; 63(4) L.F.I.

122. L.R.C. (1985), c. C-36 [ci-après L.A.C.C.]. 
lui donner une interprétation libérale et favorable aux intérêts des débiteurs. ${ }^{123}$ L'arrangement de la L.A.C.C. s'adresse autant aux créanciers ordinaires qu' aux créanciers garantis. Dans sa réforme de la proposition concordataire de la législation sur la faillite, le législateur fédéral contemporain a abondamment puisé dans la L.A.C.C., de sorte qu'on se retrouve aujourd'hui avec deux mécanismes - le concordat de la L.F.I. et l'arrangement de la L.A.C.C. — qui évoluent en parallèle et poursuivent des fins identiques. ${ }^{124}$

Si le parallélisme entre la L.A.C.C. et la procédure concordataire de la L.F.I. est indéniable, des différences significatives entre les deux régimes persistent. Ces écarts pourront expliquer ou justifier le choix de l'un ou de l'autre. Par exemple, le rejet de l'arrangement proposé en vertu de la L.A.C.C. n'entraîne pas une faillite automatique; au contraire, le refus d'accepter une proposition établie dans le cadre de la L.F.I. provoque une faillite automatique. Dans le schéma de la L.A.C.C., les tribunaux canadiens ont accueilli favorablement la doctrine américaine de la «substantive consolidation», tout en limitant son domaine par l'application de critères exigeants. ${ }^{125}$ Cette doctrine vise la liquidation ou la réorganisation concordataire du passif d'un groupe de sociétés. Elle autorise le tribunal à faire abstraction du voile corporatif et à cautionner un arrangement prévoyant la fusion des actifs et des passifs des entités juridiques distinctes formant le groupe. Les actifs du groupe se trouvent ainsi à être agrégés dans un fonds commun sur lequel l'ensemble des créances sont réalisées. Il n'est pas acquis que la doctrine de la «substantive consolidation» soit applicable aux

123. A. SERERO, «Loi sur les arrangements avec les créanciers des compagnies : considérations juridiques et pratiques», (1992) 52 Rev. du Bar. 561, 563-564; Elan Corp. c. Comitey, (1990) 1 C.B.R. (3d) 101.

124. 66 L.F.I.; 20 L.A.C.C.; il apparaît possible de combiner les deux régimes «et de se prévaloir simultanément pour partie de l'un et pour partie de l'autre» : A. BOHÉMIER, op. cit., note 107, p. 411. Le maintien de deux filières parallèles est déploré par le professeur Bohémier, qui craint la confusion et la cacophonie : A. BOHÉMIER, op. cit., note 107, p. 411. L'influent commentateur s'explique mal ce choix du législateur, dénonce l'illogisme et opine que les amendements de 1992 à la législation sur la faillite auraient dû s'accompagner de l'abrogation pure et simple de la L.A.C.C. Voir A. BOHÉMIER, op. cit., note 107, p. 410.

125. E.L. HAYES, «Substantive Consolidation Under the Companies' Creditors Arrangement Act and the Bankrupcy and Insolvency Act», [1994] C.B.L.J. 444; Northland Properties Ltd. (Re), (1988) 69 C.B.R. (N.S.) 266, 29 B.C.L.R. (2d) 257 (B.C.S.C.); Northland Properties Ltd. (Re), (1988) 73 C.B.R. (N.S.) 175 (B.C.S.C.), confirmé à [1989] 3 W.W.R. 363, 73 C.B.R. (N.S.) 195; Fairview Industries Ltd. (Re), (1991) 11 C.B.R. (3d) 43 (N.S.S.C.T.D.); Lehndorff General Partner Ltd. (Re), (1993) 17 C.B.R. (3d) 24 (Ont. Ct. (Gen. Div.)). 
propositions concordataires de la L.F.I. En témoigne l'affaire Pateman (Re), ${ }^{126}$ où la Cour du Banc de la Reine du Manitoba prononce un obiter qui traduit de sérieuses réserves quant à la possibilité de faire une proposition conjointe. ${ }^{127} \mathrm{Un}$ autre écart normatif entre les deux lois concerne les sommes dues à la Couronne. Dans le cadre d'une faillite ou d'une proposition, les réclamations ordinaires et garanties de l'État fédéral ou de l'État provincial prennent rang, en principe, comme réclamations non garanties. ${ }^{128}$ De plus, la Couronne fédérale et la Couronne provinciale sont assujetties au régime de la proposition et du concordat de la L.F.I. En ce qui concerne la L.A.C.C., le poids de la jurisprudence favorise la thèse que la Couronne n'est pas liée par la L.A.C.C., à moins qu' elle ne consente à participer au plan de redressement envisagé. ${ }^{129}$ Par ailleurs, la L.A.C.C. n'affecterait pas les droits de créancier garanti de la Couronne lorsqu'elle est intéressée à ce titre dans le patrimoine du débiteur. ${ }^{130}$ Enfin, signalons que la discrétion judiciaire est plus grande dans le processus mis en place par la L.A.C.C. que dans la procédure concordataire de la L.F.I. Le cadre de la L.A.C.C. est plus souple que celui de la L.F.I.

Pour pouvoir soumettre à ses créanciers un arrangement en vertu de la L.A.C.C., une société par actions doit non seulement être insolvable, mais aussi avoir en circulation une émission de titres d'emprunt régie par un acte de fiducie ou un instrument similaire. Par cette dernière exigence, intégrée à la loi en 1952, le législateur cherchait à restreindre le champ d'application de la L.A.C.C. aux grandes entreprises. La pratique de la L.A.C.C. n'est cependant pas allée dans ce sens. D'une part, l'acte de fiducie ${ }^{131}$ sert d'accessoire dans des techniques de financement privé employées par les petites et moyennes entreprises. ${ }^{132} \mathrm{D}$ 'autre part, les praticiens ont développé la technique de l'acte de fiducie instantané :

126. [1991] M.J. No. 221 (Q.L.); cité dans E.L. HAYES, loc. cit., note 125, 452.

127. Pateman (Re), ibid., 15. E.L. HAYES, loc. cit., note 125, 452.

128. $\quad$ 86(1) L.F.I.

129. L.J. CROZIER, «Restructuring Crown Debts under the CCAA» [1994] 23 C.B.L.J. 453; Fine's Flowers Ltd. c. Fine's Flower Ltd. (creditors), (1993) 22 C.B.R. (3d) 1, 108 D.L.R. 14th 765 (Ont. C.A.); Poulin (Gaston H.) Contractor Ltd. (Re), (1992) 10 C.B.R. (3d) 1 (Ont. Ct. (Gen. Div.)); Enterprises Jean Mercier c. Canada (Procureur général), (1992) 15 C.B.R. (3d) 35 (C.A. Québec).

130. A. BOHÉMIER, op. cit., note 107, p. 288.

131. La terminologie de l'acte de fiducie a été abolie avec le C.c.Q. et a été remplacée par l'acte d'hypothèque mettant en cause un fondé de pouvoir représentant les détenteurs de titres d'emprunt : article 2692 C.c.Q.

132. R. DEMERS, op. cit., note 90, pp. 90-93. 
une entreprise émet des titres d'emprunt en vertu d'un acte de fiducie quelques jours à peine avant d'avoir recours à la L.A.C.C. pour la seule fin d'être admissible au régime de cette loi. Les tribunaux se sont massivement exprimés en faveur de la légalité de l'acte de fiducie instantané. ${ }^{133}$

La protection de la L.A.C.C. est articulée autour de trois grandes étapes procédurales : la requête introductive, l'assemblée des créanciers et l'homologation par le tribunal. La société par actions débitrice débute le processus par la présentation d'une requête ex parte dans laquelle sont essentiellement demandés la suspension des recours et la convocation d'une assemblée des créanciers. La requête doit être accompagnée du plan d'arrangement ou d'une description de ses principaux éléments. ${ }^{134}$ Le tribunal accueillera la requête s'il est, d'une part, satisfait que les conditions d'admissibilité au régime de la L.A.C.C. sont remplies et, d'autre part, s'il est minimalement convaincu que l'entreprise possède un potentiel de viabilité économique et qu'il n'est pas improbable que le plan d'arrangement soit accepté par les créanciers. ${ }^{135}$

Il arrive que la procédure introductive prenne la forme d'une requête de sursis $^{136}$ dans laquelle le débiteur fait part de son intention de déposer dans un avenir rapproché un plan d'arrangement. ${ }^{137}$ Cette requête, si elle est accordée, opère suspension des recours. Il est obligatoire d'attacher à la requête le plan d'arrangement ou une description de ses principales composantes. ${ }^{138}$

Par la suite, le plan d'arrangement est soumis aux créanciers, réunis en assemblée. Ce plan établit une classification des créanciers faite en fonction de leur communauté d'intérêts. ${ }^{139}$ Le vote se fait par catégorie et une double majorité est requise : la majorité simple en nombre et au moins les trois-quarts en valeur. En théorie, un arrangement peut lier les créanciers des catégories qui

133. A. SERERO, loc. cit., note 123, 566-569; à titre d'illustration jurisprudentielle, voir Taché Construction Ltée c. Banque LLoyds du Canada, J.E. 90-1532 (C.S.).

134. Ibid., 578-579.

135. A. BOHÉMIER, op. cit., note 107, pp. 282-283.

136. Anglais : «Holding proposal».

137. A. BOHÉMIER, op. cit., note 107, p. 283.

138. Ibid., p. 284; Banque Laurentienne du Canada c. Groupe Bovac Ltée, J.E. 91-1398, (1991) R.L. 593 (C.A. Québec).

139. A. SERERO, loc. cit., note 123, 585. 
se sont prononcés en faveur sans pour autant contraindre ceux des catégories qui se sont exprimés contre. Il est toutefois usuel pour la société débitrice de stipuler dans le plan d'arrangement que toutes les catégories de créanciers doivent donner leur assentiment, à défaut de quoi le plan d'arrangement sera retiré. ${ }^{140}$

L'arrangement ne liera les parties que s'il est homologué par le tribunal, qui jouit à cet égard d'une discrétion étendue.

\section{E- L'arrangement de l'article 192 de la Loi sur les sociétés par actions}

En principe, les modifications structurelles d'une société doivent recevoir l'assentiment majoritaire des catégories d'actionnaires ou des porteurs de titres d'emprunt affectés. L'arrangement de l'article 192 L.S.A. déplace ce principe en permettant à une société de régime fédéral de réaliser des changements structurels majeurs sous contrôle judiciaire. C'est le 3e paragraphe de l'article 192 L.S.A. qui est la pierre angulaire du mécanisme de l'arrangement

\section{«(3) [Demande d'approbation au tribunal]}

Lorsque la société, qui n'est pas insolvable, n'est pas en mesure d'opérer, en vertu d'une autre disposition de la présente loi, une modification de structure équivalente à un arrangement, elle peut demander au tribunal d'approuver, par ordonnance, l'arrangement qu'elle propose.»

Le tribunal jouit d'une discrétion étendue. Il se trouve à piloter le processus interne par lequel les changements structurels se réaliseront. Il a notamment le pouvoir de demander que les détenteurs de valeurs mobilières de la société approuvent l'arrangement, selon des modalités qu'il aura auparavant fixées.

L'article 192(1) L.S.A. dresse une liste non limitative des modifications de structure envisagées par l'arrangement. Parmi celles-ci, on retrouve la fusion, le fractionnement de l'activité commerciale, la cession de la totalité ou de la quasi-totalité des biens. Instrument souple et à grande portée, 
l'arrangement peut servir de cadre procédural à la restructuration d'une entreprise en difficulté, en particulier lorsque l'opération est complexe et comporte plusieurs ordres de changements structuraux. On l'a ainsi utilisé en 1988 dans le sauvetage de l'importante société pétrolière Dome Petroleum Limited. ${ }^{141}$

Pour se prévaloir de la procédure de l'arrangement, une société ne doit pas être dans un état d'insolvabilité. Elle doit aussi convaincre le tribunal qu'elle n'est pas en mesure d'opérer, en vertu d'une autre disposition de la L.S.A., une modification de structure équivalente à un arrangement. À la lumière de la jurisprudence, «ne pas être en mesure» ne signifie pas «impossible» mais «difficile». ${ }^{142}$

L'arrangement présente l'avantage de s'appliquer à la fois à l'endettement et aux capitaux propres de l'entreprise. L'ordonnance du tribunal approuvant l'arrangement peut lier non seulement les détenteurs de titres de participation mais aussi les détenteurs de titres d'emprunt. L'arrangement n'atteint cependant pas les créanciers dont les droits ne sont pas constatés par des valeurs mobilières, ce qui limite l'efficacité de la procédure employée dans une optique de redressement. Il est possible de déployer l'arrangement de l'article 192 L.S.A. conjointement avec le mécanisme de la L.A.C.C. ${ }^{143}$

\section{F- L’action privilégiée dite «de détresse»}

L'émission d'actions privilégiées est le mode classique par lequel la dette d'une société est convertie en capitaux propres. ${ }^{144}$ Parmi les techniques déployées, une avenue particulièrement intéressante pour l'entreprise en difficulté est la conversion d'une dette en actions privilégiées à court terme dites «de détresse». ${ }^{145}$ L'avantage pour la société débitrice tient à ce que les

141. S.B. SCOTT, T.O. BUCKLEY et A. HARRISON, «The Arrangement Procedure Under Section 192 of the Canada Business Corporations Act and the Reorganization of Dome Petroleum Limited», [1990] 16 C.B.L.J. 296.

142. Ibid., 304.

143. 20 L.A.C.C.

144. D.A. BALL, «Corporate Aspects of Reorganization and Refinancing», (1984) 1 Nat. Ins. Rev. 84, 93.

145. Anglais : «Distress Preferred Shares». 
dividendes versés sur ces titres sont non imposables. ${ }^{146}$ Le créancier, détenteur des actions privilégiées, est donc prêt à accepter des dividendes moins élevés que les intérêts sur sa créance, ce qui soulage d'autant la société débitrice. Les actions privilégiées «de détresse» sont émises pour une période maximale de 5 ans. L'émission doit se faire à la suite du défaut de l'emprunteur relativement à la dette faisant l'objet de la conversion.

Dans l'opération de conversion typique, le prêteur cède sa créance en souffrance à une filiale de l'emprunteur, constituée spécialement pour ces fins. L'acquisition est financée par un «prêt d'un jour», ${ }^{147}$ octroyé par le prêteur. Puis, le prêteur souscrit des actions privilégiées «de détresse» émises par la filiale. L'apport relatif à la souscription est constitué par le remboursement du «prêt d'un jour». La filiale, qui est le nouveau créancier de l'emprunteur, renonce à ses paiements d'intérêt, en contrepartie de quoi l'emprunteur s'engage à fournir à sa filiale les fonds pour financer le paiement des dividendes sur les actions privilégiées «de détresse». En plus, l'emprunteur convient d'injecter du capital dans sa filiale de manière à lui permettre de racheter à échéance les actions privilégiées. ${ }^{148}$

\section{CONCLUSION}

L'endettement de l'entreprise baigne dans une singulière mixture juridique où se côtoient classicisme et courants novateurs. Le classicisme ressort du rôle prépondérant de la dynamique de marché dans la fonction régulatrice du niveau d'endettement des entreprises ou dans l'attachement des tribunaux à la liberté contractuelle et au principe de la force obligatoire des contrats. En particulier, cet attachement joue sur plusieurs gammes. Il se manifeste dans les réticences judiciaires à l'égard des conceptions françaises de la responsabilité professionnelle du banquier. Il s'exprime par une tendance lourde de non-interventionnisme judiciaire et législatif au chapitre de la définition des modalités de l'endettement. Il anime la dialectique qui produit la

146. J.A.H. GARNER, «Refinancing Instruments for Financially Distressed Companies», (1993) 12 National Banking Law Review 75, 82.

147. Anglais : «Daylight loan».

148. Cette description d'une opération-type est tirée de E.A. FISHER, loc. cit., note 23, 23; pour une illustration jurisprudentielle de cette technique, voir Pioneer Distributions Ltd. c. Bank of Montreal, (1994) 97 B.C.L.R. (2d) 143. 
qualification de dette ou de fonds propres, dialectique marquée par la recherche de l'intention commune des parties. Classicisme aussi, dans la froide réserve ressentie à l'égard de ces doctrines d'inspiration américaine, pourtant séduisantes par leur petit côté inattendu, irrévérencieux, voire contre-culturel. Classicisme, somme toute, dans la spontanéité, la promptitude et le naturel avec lesquels le pouvoir judiciaire se tourne vers les institutions juridiques du libéralisme économique lorsqu'il compose avec la problématique de l'endettement de l'entreprise. ${ }^{149}$

Mais innovation, et audace, dans le déploiement imaginatif du recours pour oppression de l'actionnaire ou l'utilisation astucieuse de la procédure de l'arrangement de la L.S.A. Innovation, et modernité, dans la volonté énergique et clairvoyante du législateur québécois d'implanter un système de sûretés qui reconnaît la spécificité de l'entreprise et la soutient dans ses aspirations. Innovation, et générosité de vues, dans le recul des frontières de la théorie de l'abus des droits contractuels appliquée à la réalisation des sûretés, dans la largesse de l'interprétation et de l'application de la L.A.C.C. ou, encore, dans l'élaboration du mécanisme de la proposition concordataire de la nouvelle législation sur la faillite et l'insolvabilité.

149. Ainsi, le professeur Antaki écrivait en 1982 : «Les tribunaux du Québec adhèrent encore généralement au libéralisme économique le plus absolu, au moins en ce qui concerne le droit des affaires». Voir N. ANTAKI, «Rapport général», dans La survie des entreprises privées en difficulté, op. cit., note 21, p. 316. L'affirmation, nous semble-t-il, est encore d'actualité; il faut toutefois reconnaître qu'elle a été relativisée par des développements jurisprudentiels récents, comme par exemple la décision Houle c. Banque nationale du Canada, précitée, note 96. 